\title{
Polarization data from SCIAMACHY limb backscatter observations compared to vector radiative transfer model simulations
}

\author{
P. Liebing, K. Bramstedt, S. Noël, V. Rozanov, H. Bovensmann, and J. P. Burrows \\ University of Bremen, Institute of Environmental Physics, P.O. Box 33 04 40, 28334 Bremen, Germany \\ Correspondence to: P. Liebing (patricia.liebing@iup.physik.uni-bremen.de)
}

Received: 9 February 2012 - Published in Atmos. Meas. Tech. Discuss.: 15 March 2012

Revised: 16 April 2013 - Accepted: 29 April 2013 - Published: 5 June 2013

\begin{abstract}
SCIAMACHY is a passive imaging spectrometer onboard ENVISAT designed to obtain trace gas abundances from measured radiances and irradiances in the UV to SWIR range in nadir-, limb- and occultation-viewing modes. Its grating spectrometer introduces a substantial sensitivity to the polarization of the incoming light with nonnegligible effects on the radiometric calibration. To be able to correct for the polarization sensitivity, SCIAMACHY utilizes broadband Polarization Measurement Devices (PMDs). While for the nadir-viewing mode the measured atmospheric polarization has been validated against POLDER data (Tilstra and Stammes, 2007, 2010), a similar validation study regarding the limb-viewing mode has not yet been performed. This paper aims at an assessment of the quality of the SCIAMACHY limb polarization data. Since limb polarization measurements by other air/spaceborne instruments in the spectral range of SCIAMACHY are not available, a comparison with radiative transfer simulations by SCIATRAN V3.1 (Rozanov et al., 2013) using a wide range of atmospheric parameters is performed. SCIATRAN is a vector radiative transfer model (VRTM) capable of performing calculations of the multiply scattered radiance in a spherically symmetric atmosphere.

The study shows that the limb polarization data exhibit a large time-dependent bias that decreases with wavelength. Possible reasons for this bias are a still unknown combination of insufficient accuracy or inconsistencies of the on-ground calibration data, scan mirror degradation and stress induced changes of the polarization response of components inside the optical bench of the instrument. It is shown that it should in principle be feasible to recalibrate the effective polarization sensitivity of the instrument using the in-flight data and VRTM simulations.
\end{abstract}

\section{Introduction}

SCIAMACHY (SCanning Imaging Absorption spectroMeter for Atmospheric CHartographY) is in a polar sunsynchronous orbit onboard ESA's ENVISAT platform. It obtains spectra of the solar radiance as it is reflected, scattered or transmitted by the Earth in limb-, nadir- as well as solar and lunar occultation-viewing modes by means of a grating spectrometer with moderate spectral resolution between 0.2 and $1.5 \mathrm{~nm}$ (Bovensmann et al., 1999). Its spectral range covers the region between 240 and $1700 \mathrm{~nm}$ as well as two bands around 2 and $2.4 \mu \mathrm{m}$. In a typical orbit, limb and nadir scans are alternated such that their footprints overlap. Limb scans are typically performed in 30 steps of about $3.3 \mathrm{~km}$ from just below the horizon to about $93 \mathrm{~km}$, with a total horizontal scan size of about $960 \mathrm{~km}$. In each horizontal scan 4 measurements are taken, resulting in an effective field of view (FoV) of about $260 \mathrm{~km}$ across track and $2.6 \mathrm{~km}$ vertically at the tangent point. The limb scans performed on the day side of each orbit cover a range of solar zenith angles between 20 and $90^{\circ}$ and relative azimuth angles between 20 and $160^{\circ}$. An overview of the instrument design and its features is given in Gottwald and Bovensmann (2011).

The sensitivity of the instrument response to the polarization state of the light entering the instrument's FoV, i.e., its polarization sensitivity, originates mainly from the spectrometer grating in the main part of the science channels. At the channel boundaries, large and spectrally highly variable polarization effects are generated by the dichroic mirrors. The polarization sensitivity is further modified by the predisperser prism and the scanning mirrors. In the limb mode its magnitude ranges up to 0.4 with some particular spectral features mainly in the UV region. This means that the 
instrument throughput can be up to $40 \% \cdot P$ different for light with a degree of polarization $P$ compared to unpolarized light. The polarization of the scattered sunlight follows a generic pattern along the (sun-synchronous) orbit given by the specific scattering geometry of each limb scan. Its variability increases with wavelength due to the increasing influence of scattering on aerosols, cloud droplets and the surface compared to pure Rayleigh scattering on molecules. Radiometric errors arising from uncorrected polarization sensitivity could be as high as $20 \%$ and lead to systematic errors depending on latitude and season, directly in the reflectance measurements but possibly also indirectly in derived products such as trace gas or aerosol concentrations.

The instrument's polarization sensitivity was measured in a dedicated on-ground calibration campaign. To be able to correct the measured signals for the polarization-dependent throughput, the polarization is measured by the so-called Polarization Measurement Devices (PMDs) in 5 different wavelength bands whose average wavelength roughly matches with the central wavelength in SCIAMACHY channels 2 to 6 . In this way it is in principle possible to determine a smoothed polarization spectrum between 300 and $1700 \mathrm{~nm}^{1}$. It is not possible to obtain measurements of spectral features in the polarization arising from strong trace gas absorption where the photon light path is significantly altered, or from Raman scattering around Fraunhofer lines. The PMDs are sampling detectors with high sensitivity to light polarized parallel to the instrument's entrance slit. An additional PMD is particularly sensitive to $45^{\circ}$ polarized light in the same spectral range as PMD 4 around $850 \mathrm{~nm}$.

The polarization measurements benefit not only the accurate radiometric calibration of SCIAMACHY radiance spectra, they could also provide valuable information on microphysical parameters of aerosols and clouds (Lebsock et al., 2007). Radiance data alone, in particular if only a single viewing direction per scanned air volume is available, can usually not resolve the ambiguities between effects of the surface albedo, the aerosol concentration and its microscopic properties (Kokhanovsky et al., 2007). The addition of polarization information could in principle provide constraints on different aerosol models. This is in general true for both nadir- and limb-viewing modes. Global sets of dedicated multispectral and multiview nadir polarization measurements are available from the POLDER instruments (Deschamps et al., 1994) onboard ADEOS, ADEOS-II and PARASOL (Bréon et al., 2002). GOME (Burrows et al., 1999) and GOME-2 (Munro et al., 2006) measure the nadir polarization in a similar manner to SCIAMACHY (Krijger et al., 2004; Callies et al., 2002). CALIOP on CALIPSO pro-

\footnotetext{
${ }^{1} \mathrm{~A}$ sixth PMD is installed for the $2.0<\lambda<2.4 \mu \mathrm{m}$ range; however, the corresponding SCIAMACHY pixel detectors cover only one third of its wavelength range. Because of this and due to hardware problems in both the PMD and the pixels detectors, polarization values obtained from PMD 6 are therefore highly unreliable.
}

vides lidar depolarization measurements at $532 \mathrm{~nm}$ with good height resolution but small spatial coverage (Winker et al., 2009). Aside from SCIAMACHY, limb polarization measurements are only available from a number of aircraft missions (McLinden et al., 1999). Indirect measurements in the UV region have been performed as part of $\mathrm{O}_{3}$ retrievals from OSIRIS spectra (McLinden et al., 2004). SCIAMACHY, however, has the unique potential to provide the only contiguous and global limb polarization profile data available, now spanning almost 10 years.

In light of this it is vital to validate the SCIAMACHY limb polarization data. Due to the lack of both polarized internal calibration sources and independent measurements, the validation has to be performed against a radiative transfer model capable of simulating the Stokes vector of the limb-scattered intensity in a spherical atmosphere. This paper presents a comparison of limb polarization data from SCIAMACHY obtained between 2004 and 2010 with SCIATRAN (version 3.1) simulations for a wide range of atmospheric scenarios. An investigation of possible instrumental and theoretical error sources has been performed. The possibility of using model simulations for in-flight calibration of the polarization sensitivity will also be discussed, and first results will be shown.

In Sect. 2, the measurement and calibration methods relevant for the determination of the polarization are introduced. The selection of the data set used for this study is motivated. Section 3 gives a brief overview of SCIATRAN and the setup for the simulations. A comparison of the simulations and the data on a statistical basis is presented in Sect. 4 . An investigation of possible error sources and a discussion of options for the in-flight calibration and monitoring are discussed together with first results in Sect. 5.

\section{Measurement method and data selection}

\subsection{General calibration and measurement concept}

The algorithm to determine the polarization makes use of the Mueller matrix formalism. Sunlight reflected and scattered into the instrument FoV can be described by the components of a Stokes vector:

$\boldsymbol{I}=\left[\begin{array}{c}I \\ Q \\ U \\ V\end{array}\right]$,

where $I$ is the total intensity in photons $\mathrm{s}^{-1} \mathrm{sr}^{-1} \mathrm{~nm}^{-1} \mathrm{~cm}^{-2}$ and

$Q=I_{\|}-I_{\perp}, \quad U=I_{45^{\circ}}-I_{-45^{\circ}}$.

The linear polarization components $Q$ and $U$ are defined along the $\mathrm{x}$-axis and along the $45^{\circ}$ direction in a given reference coordinate system, respectively, and $V$ is the circular polarization. 
The end-to-end Mueller matrix $\mathbf{M}$ describes the instrument response to each of the Stokes vector components:

$S_{\mathrm{det}}=[\mathbf{M} \cdot \boldsymbol{I}]_{0}=I M_{11}\left(1+\frac{M_{12}}{M_{11}} \frac{Q}{I}+\frac{M_{13}}{M_{11}} \frac{U}{I}\right)$.

The detector signal $S_{\text {det }}=S_{\text {raw }}-S_{\text {offset }}$ (i.e., the raw ADC signal corrected for all additive contributions such as pedestal and dark current) is the first component of the resulting Stokes vector; therefore, in Eq. (3) only the first row of the Mueller matrix is relevant. The circular component of the atmospheric polarization is negligibly small (Hansen and Travis, 1974) such that the detected signal can be described in terms of the total intensity, the absolute radiance sensitivity $M_{11}$, the relative polarization sensitivities

$\mu_{i}=\frac{M_{1 i}}{M_{11}}, \quad i=2,3$

and the degrees of polarization of the second and third Stokes components: $q=\frac{Q}{I}$ and $u=\frac{U}{I}$.

The wavelength-dependent Mueller matrix includes the response of both the Optical Bench Module (OBM) and the scanner module. As in each SCIAMACHY measurement mode a different setup of the scanner module is used, the Mueller matrix depends on the measurement mode and the involved scan angles. For limb measurements, the Mueller matrix includes the effects of the elevation scan module (ESM) as well as the azimuth scan module (ASM) mirrors. The OBM comprises all components behind the scanner module. The Mueller matrix elements (MMEs) are derived from on-ground measurements of the polarization sensitivity (the so-called "Greek" calibration key data).

Every polarization measurement requires the determination of at least two independent observables of the same light beam. The measurement approach taken for SCIAMACHY is to split the incoming light into two beams with known fraction and measure the signal in two detectors with different, known polarization sensitivity. The predisperser prism behind the entrance slit to the telescope generates one fully polarized beam directed towards the PMDs and one beam that is further processed by the spectrometer and recorded by the science pixel detectors. The PMDs sample the signal at a frequency of $40 \mathrm{~Hz}$. The PMD signals have to be synchronized to the integrated signal of the detector pixels and integrated over the exposure time of the corresponding science detectors. In total, seven PMDs are installed, where PMDs 1-6 are mostly sensitive to $Q$ and PMD 7 is sensitive to $U$. Table 1 lists the PMDs with their spectral range and average wavelengths for typical limb spectra. PMD 6, which is sensitive in the $2.0<\lambda<2.4 \mu \mathrm{m}$ range, will not be discussed here because its polarization values are not meaningful due to lack of corresponding science detector coverage. A more detailed description of the calibration concept can be found in Gottwald and Bovensmann (2011).
Table 1. SCIAMACHY PMDs, their spectral range, average wavelength, corresponding SCIAMACHY science channel and average polarization sensitivities for limb measurements.

\begin{tabular}{rrrcrr}
\hline PMD & $\begin{array}{r}\text { Range } \\
(\mathrm{nm})\end{array}$ & $\begin{array}{r}\langle\lambda\rangle \\
(\mathrm{nm})\end{array}$ & Chan. & $\left\langle\mu_{2}\right\rangle$ & $\left\langle\mu_{3}\right\rangle$ \\
\hline 1 & $310-385$ & 352 & 2 & 0.981 & -0.108 \\
2 & $440-530$ & 484 & 3 & 0.987 & -0.076 \\
3 & $600-710$ & 656 & 4 & 0.994 & -0.060 \\
4 & $780-920$ & 852 & 5 & 0.994 & -0.067 \\
5 & $1450-1700$ & 1570 & 6 & 0.996 & -0.004 \\
7 & $780-920$ & 854 & 5 & -0.044 & 0.991 \\
\hline
\end{tabular}

\subsection{Determination of polarization values}

The operational polarization algorithm makes use of the fact that the intensities corresponding to the integrated signals of the science pixels and the PMDs have to be the same. The signals of the $i$-th pixel of the science channel and the $i$-th (virtual) pixel of the PMD according to Eq. (3) are:

$S_{i}^{\mathrm{D}(\mathrm{P})}=I M_{11, i}^{\mathrm{D}(\mathrm{P})}\left(1+\mu_{2, i}^{\mathrm{D}(\mathrm{P})} q+\mu_{3, i}^{\mathrm{D}(\mathrm{P})} u\right)$.

The integrated signals of the science channel over the spectral range of the PMD and PMD signal can then be related to each other by

$\mathrm{IB} \cdot S^{\mathrm{P}}=\sum_{i} S_{i}^{\mathrm{D}} M_{1, i}^{\mathrm{PD}} \frac{1+\mu_{2, i}^{\mathrm{P}} q+\mu_{3, i}^{\mathrm{P}} u}{1+\mu_{2, i}^{\mathrm{D}} q+\mu_{3, i}^{\mathrm{D}} u}, \quad$ with

$M_{1, i}^{\mathrm{PD}}=\frac{M_{11, i}^{\mathrm{P}}}{M_{11, i}^{\mathrm{D}}}$.

The superscripts P and D refer to PMD and pixel detectors, respectively, and the sum is over all pixels from the start to the end of the PMD spectral range. The sum on the righthand side of Eq. (6) is called virtual sum, and Eq. (6) is called virtual sum equation. The scale factor IB is the so-called inband signal and should account for initial calibration errors in the radiance response ratio $M_{1}^{\mathrm{PD}}$, for gaps (due to bad pixels) or cutoffs in the pixel detector range and for degradation effects. It is determined from solar reference measurements, which are performed daily, and ensures that for unpolarized light $(q=0, u=0)$ the scaled PMD signal is equal to the virtual sum. However, since the spectral shape of the solar irradiance in the reference measurements is very different from that of the limb and nadir Earth shine spectra, and since each measurement mode uses a different scanner configuration, the in-band signal may actually cause a constant polarization bias.

The virtual sum equation is solved numerically for $q$ assuming that it is constant over the PMD spectral range. The result therefore has to be regarded as an effective value for $q$ 
over the corresponding wavelength range. As the two measurements allow only the determination of one polarization component, the assumption

$u / q=$ const. $=u_{\mathrm{SS}} / q_{\mathrm{SS}}$

is made, with $u_{\mathrm{SS}} / q_{\mathrm{SS}}$ being the ratio of $u$ and $q$ for single Rayleigh scattering. This assumption was justified by model studies representative for nadir conditions (Schutgens et al., 2004). SCIATRAN simulations performed for this study showed that the assumption is well justified above $500 \mathrm{~nm}$ in the nadir mode. In the UV between 300 and $400 \mathrm{~nm}$, the ratio is not constant, although in most cases the resulting errors on $u$ are below 0.1. For the limb mode, SCIATRAN simulations in general indicate a higher variability of the $u / q$ ratio with consequent errors on $u$ of up to 0.2 even at visible wavelengths. However, as discussed below in Sect. 3.1, intrinsic model errors in SCIATRAN currently inhibit quantitative conclusions on this issue. In the case of very small $\left|q_{\mathrm{SS}}\right|, u$ is assumed to be $c \cdot u_{\mathrm{SS}}$ with $c$ a factor, depending on mode and wavelength, determined from model studies (Slijkhuis, 2008). The on-ground key data suggest that the PMD sensitivity to $u$ given by $\mu_{3}^{\mathrm{P}}$ is relatively small, except for PMD 1 (see Table 1). Errors related to the assumption on $u$ are therefore usually also small. However, this is only true as long as $\left|\mu_{2}^{\mathrm{P}} q_{\mathrm{SS}}\right| \gg\left|\mu_{3}^{\mathrm{P}} u_{\mathrm{SS}}\right|$. If both terms are roughly equal and nearly cancel each other, a value of the virtual sum around 1 will be misinterpreted as a small value of $q$ and $u$. This may result in large errors of the polarization values as well as the polarization correction term. This issue will be further discussed in Sect. 5.1.

The Stokes vector and Mueller matrix need to be defined in a common reference frame. The current operational processor (version 7.03) uses two separate frame definitions for the internal processing and the Level 1 product values. The internal frame is defined with regard to the entrance slit such that $q$ is positive when the polarization is parallel to it and $u$ is positive when the polarization is along a $45^{\circ}$ clockwise rotation (looking into the instrument at the location of the spectrometer slit) from the parallel direction. The atmospheric frame definition in the Level 1 product uses the local meridional plane, which is the plane spanned between the line-ofsight and the local zenith. Positive $q$ is the polarization lying in this plane and therefore in the scanning direction of the SCIAMACHY FoV. Positive $u$ is again defined for a clockwise rotation from the parallel direction when looking in the travel direction of the light. An illustration of this coordinate frame definition for the nadir mode can be found in Fig. 5.5 of Gottwald and Bovensmann (2011).

The conversion between the internal frame and the atmospheric frame needs to take into account the $90^{\circ}$ rotation between the scanning plane and the entrance slit as well as the scan mirror reflections. For the limb mode involving the ASM and ESM mirrors, the conversion can be summarized by

$q_{\text {atmos }}=-q_{\text {internal }} \quad$ and
$u_{\text {atmos }}=-u_{\text {internal }}$.

It is important that the coordinate frame definitions are used consistently throughout the algorithm chain starting from the determination of the MMEs up to the retrieval of the polarization values from the measurements. This has proved to be exceptionally difficult for the $45^{\circ}$ polarization or, rather, the contribution of $\mu_{3}^{\mathrm{P}} u$ to the PMD signal. In-flight polarization data from PMD 1 and PMD 7, where this contribution is largest, indicate that in the currently used version of the calibration key data, the sign of $\mu_{3}^{\mathrm{P}} u$ for limb is correct for PMD 1 but wrong for PMD 7. In this analysis, the sign of $\mu_{3}^{\mathrm{P}}$ of PMD 7 was therefore reversed to obtain consistency with the other PMDs ${ }^{2}$.

\subsection{Data selection and processing}

The data set used in this study was selected so as to facilitate the comparison with the model data. First, 1 measurement orbit was chosen arbitrarily, from which 22 limb measurement sets (called states) with 4 profiles each and with a solar zenith angle (SZA) lower than $80^{\circ}$ on the descending node of the orbit were selected. This reference orbit is orbit 33750 from 13 August, 2008. The measurement time lines of SCIAMACHY cause a recurrence of the exact same viewing geometries of some of these 22 states in about every other orbit in the period between the 9 and 20 August for each year between 2004 and 2010, and also in a smaller set of data from mid-April of these years. Altogether, about 550 orbits were found where a minimum of 8 states matched the viewing geometries of the corresponding reference states. A state was called a match with the reference state if the solar zenith angles agreed within $0.1^{\circ}$ and the relative azimuth angles within $1^{\circ}$ for all tangent height steps. Before 2004 and after 2010 there are no matching states because the nominal execution of limb states was different then. Note that no particular requirement was imposed on the location of the tangent point except for the exclusion of the Southern Atlantic Anomaly (SAA) region. Effectively this means that the viewing geometry corresponding to any given reference state is evenly distributed over all longitudes but covers only a very narrow latitude band. The average statistics for each reference state per year varies between 20 and 50 for the August data. Figure 1 shows the latitudes, SZA and single scattering angles $\theta$ for the described data set versus the state index of the reference orbit. Note that no explicit requirement was imposed on the tangent height itself, in fact it varies randomly by a few hundred meters at each step. The selected data set allows a statistical analysis of data with the same measurement

\footnotetext{
${ }^{2}$ The polarization value from PMD 7 is not used operationally; the sign error in $u$ therefore does not enter the radiometric calibration.
} 

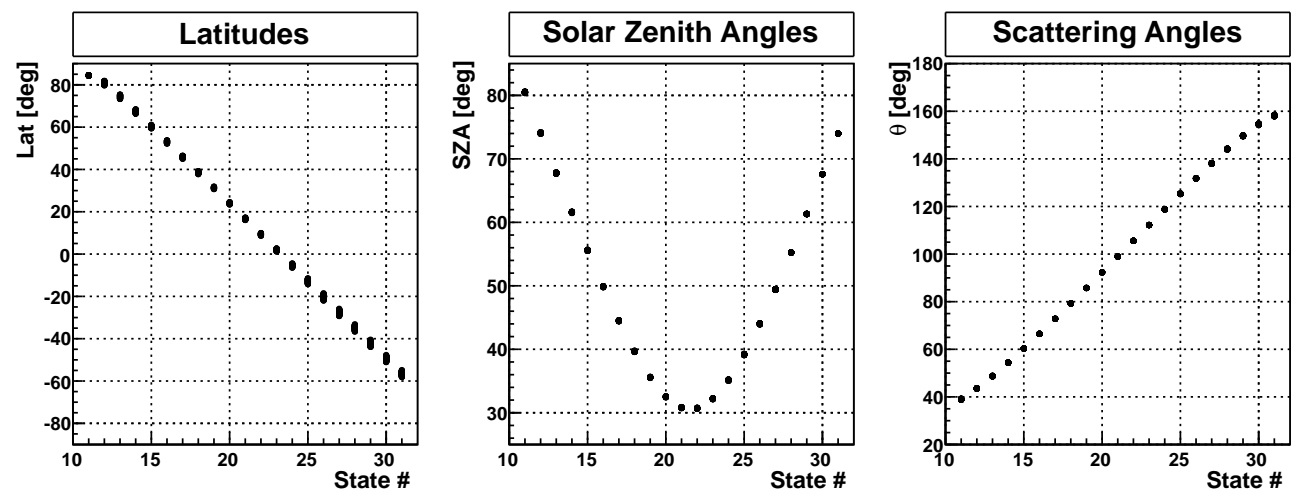

Fig. 1. Latitudes (left panel), SZA (middle panel) and single scattering angles for the data set used in this study vs. the state counter of the reference orbit 33750 (see text).

configurations but different atmospheric and surface conditions while reducing the number of different states to be simulated by SCIATRAN to about 20. Version 7.03/7.04 of the operational Level 1b (L1B) data product was used to obtain the pixel and PMD signals, viewing angles, geolocation information and auxiliary information from which the polarization values were then calculated. The algorithm applied is similar to, but not exactly the same as the one applied in the operational Level $0-1$ processing. The changes to the polarization algorithm compared to the operational processor are described in Appendix A. Below $30 \mathrm{~km}$ the differences in the results between the operational and this algorithm are very small.

The radiometrically calibrated intensities were extracted from the L1B data using the scia_nl1 tool (van Hees, 2012) for the application of the radiometric calibration. The applied calibration steps include the analog offset and limb dark current subtractions, nonlinearity correction in channel 6 , internal stray light correction, radiometric calibration with polarization correction and degradation correction. The memory effect correction in channels 1 to 5 was not applied.

\section{SCIATRAN simulations}

\subsection{SCIATRAN}

The specific viewing and scattering geometries of limb measurements require the solution of the vector radiative transfer equation (VRTE) in a spherical atmosphere to simulate the the radiance and polarization as measured by SCIAMACHY. For this study simulations are performed using SCIATRAN V3.1 (Rozanov et al., 2013). In SCIATRAN, the solution of the VRTE at each point along the line of sight is achieved by decomposing the Stokes vector of the diffuse radiation and the scattering matrix in each atmospheric layer into a Fourier series and then solving the equation for each Fourier component using the discrete ordinates technique. The single scattering contribution is integrated for each (spherical) at- mospheric layer along the line of sight. To compute the multiple scattering contribution, the combined differentialintegral (CDI) approach is applied (Rozanov et al., 2000). In this approach, the multiple scattering source function is calculated at a number of discrete points corresponding to different solar zenith angles along the line of sight. At each of these points the diffuse radiation field is approximated by that of a pseudospherical atmosphere. This means that the transmission of the incident (solar) radiation is calculated within a spherically layered atmosphere, while the scattered radiation is calculated within a plane parallel atmosphere. The results for each discrete point are subsequently interpolated and integrated along the line of sight, in this way properly regarding the curvature of the surface and atmospheric layers. In principle it is possible to repeat this calculation iteratively to arrive at a more accurate estimate of the top-of-atmosphere (TOA) reflectance (Rozanov et al., 2001). In SCIATRAN, this option is only available for the scalar mode; in the vector mode it has not yet been implemented. Also, because of the computational effort it would not be feasible to run extensive model studies as the one presented here with the iterative scheme.

The vertical inhomogeneity of the atmosphere is modeled by dividing the atmosphere into homogeneous layers on a user-defined grid. Input profiles of pressure, temperature and, if desired, trace gas abundances and aerosol concentrations are interpolated to the middle between grid points to obtain a smoothly varying profile. Atmospheric refraction can be taken into account as well as the integration of the radiance over a vertical FoV. The surface reflection is modeled by a Lambertian albedo or by a bidirectional reflectance distribution function (BRDF). The input to SCIATRAN is a set of line-of-sight and solar zenith angles and the relative azimuth angles between the line of sight and the solar direction at the TOA. The output is the Stokes vector at the TOA in units of radiance or solar irradiance.

For the scalar mode, a comparison of the CDI approximation with the fully iterated solution showed considerable 
errors of a few percent in the calculated reflectance above $30 \mathrm{~km}$ (Rozanov et al., 2002). An ongoing comparison between SCIATRAN in the vector mode and two Monte Carlo VRTMs, SIRO (Oikarinen et al., 1999) and MYSTIC (Emde et al., 2010; Mayer, 2009), revealed that not only the reflectance suffers from inaccuracies but also and in particular the polarization. The relative errors of $q$ can be larger than $10 \%$ on occasions, even at tangent heights as low as $20 \mathrm{~km}$. Inaccuracies generally increase with tangent height and with increasing contribution from multiple scattering or scattering at the surface. These results do not invalidate any of the conclusions drawn here on the quality of the SCIAMACHY data as shown below; however, they demand further improvement of the model.

\subsection{Scenarios}

The TOA reflectance at any given wavelength depends on a variety of atmospheric and surface parameters, most of which are generally not or only approximately known. This leaves two options for a quality assessment as this one. One could pick a few data points at particular measurement times and locations for which the atmospheric composition is very well known; for instance, a cloudless scene over the ocean far from anthropogenic or natural pollution sources. This would yield a small, and likely highly biased, data set. With the large FoV of SCIAMACHY it will be very difficult, though, to positively exclude the presence of clouds. In addition, uncertainties in the description of the BRDF and optical aerosol properties arise. A study along the lines of this idea was performed with the result that even with strict selection filters yielding not more than a handful of data points, the variability of the measured reflectance between the selected data points is too large to distinguish between radiometric calibration errors and model parameter uncertainties. Typically, the measured intensities matched modeled ones to within $20 \%$ at tangent heights below $30 \mathrm{~km}$. The small number of data points did not allow for a systematic investigation of the polarization values.

The second approach, which will be followed here, is to generate simulations spanning a large parameter space that could in principle accommodate most of the situations, and then study the statistical behavior of the data with respect to this parameter set. This approach can help identify biases, but again will not help in identifying calibration errors on a few-percent level.

SCIATRAN simulations were performed for the first profile of 20 out of the $22 \mathrm{limb}$ states $^{3}$ with a large number of different atmospheric parameter settings. The surface reflectance was simulated with a Lambertian albedo between 0 and 1 . The aerosol profile was divided into three layers (boundary layer, tropospheric and stratospheric aerosol) with

\footnotetext{
${ }^{3}$ There were errors in the simulation of two states, which is why they are not used here.
}

different types of aerosol and different aerosol loads. The shape of the profile in each layer was fixed, while the aerosol optical depth (AOD) was varied. Any combination of layer AOD and aerosol type within the first three layers was allowed. The aerosol types used for each layer are mixtures of the basic types recommended in the WMO report (Deepak and Gerber, 1983; Bolle, 1986). Appendix B lists the details of the aerosol types and profiles used. All aerosol types were assumed to consist of spherical particles, and the corresponding phase matrix was calculated using Mie theory.

Pressure and temperature profiles were fixed to the USStandard scenario (COESA, 1976). Every major trace gas absorber relevant for the considered spectral range (300$1700 \mathrm{~nm}$ ) was included in the simulation with a fixed profile using a climatological data base similar to that described in Haley et al. (2004) and McLinden et al. (2010). Absorption cross sections were taken from the HITRAN 2004 data base for line absorbers (Rothman et al., 2005) $\left(\mathrm{O}_{2}, \mathrm{H}_{2} \mathrm{O}, \mathrm{CO}_{2}\right)$ and from measurements by the SCIAMACHY PFM satellite spectrometer for $\mathrm{O}_{3}$ and $\mathrm{NO}_{2}$ (Bogumil et al., 2003). The impact of density and absorber profile variations on the simulated radiances and polarization has been studied; results are discussed in Appendix B.

It is obvious that not all the possible combinations of albedo, aerosol and atmospheric species can be considered to be realistic assumptions for the data sample studied here. In particular, some of the aerosol scenarios are extremely exaggerated compared to typically prevailing conditions. However, the definition of extreme scenarios may help in understanding the limits within which measurements are expected to fall. It is later possible to select specific scenarios that match the data to a first approximation and conduct a more refined comparison between data and model.

In addition to the described main simulation data set, two smaller sets were generated as control samples to allow an assessment of some of the model dependence. Each of these comprises a subset of the limb states and of the aerosol and albedo scenarios of the main sample. In addition, in the first set, the aerosol content in the fourth (mesospheric) aerosol layer is significantly increased and varied. In the other set, clouds of different types, cloud top height and optical depth were simulated. Both sets serve as control samples to study the model dependence of this comparison.

\subsection{Simulation of SCIAMACHY reflectances and polarization values}

To compute the polarization as measured by SCIAMACHY, it would in principle be necessary to calculate the Stokes vector for the full wavelength range covered by the PMD at a high resolution, convolute to the instrument's spectral resolution and then average the spectra weighted with the PMD efficiency. As SCIATRAN runs relatively slow in the vector mode, in particular with the spherical geometry, the simulations of all of the different scenarios described above with 

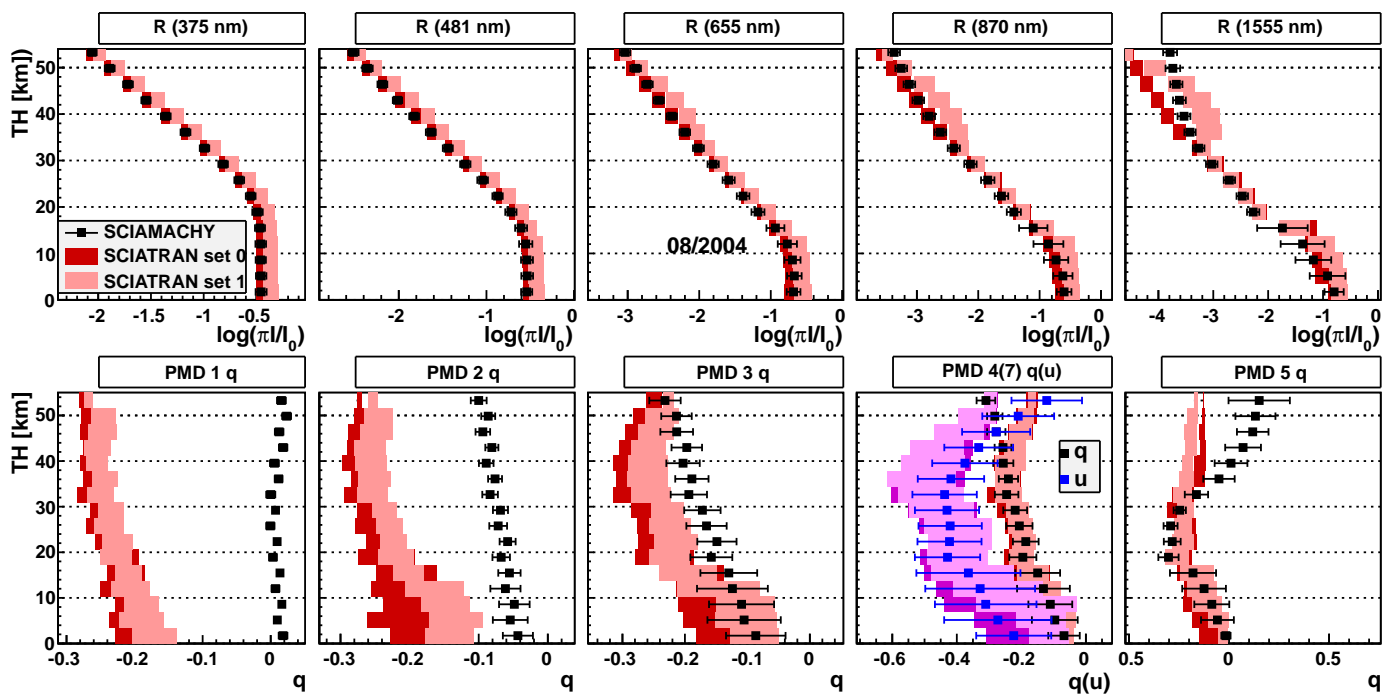

Fig. 2. Reflectance (top panel) and polarization profiles (bottom panel) for SCIAMACHY data (points) and SCIATRAN (boxes, red for $q$, magenta for $u$ ); the shading indicates different simulation sets (see text for detail). The data set is for the reference state $20\left(\theta=92^{\circ}\right.$, $\mathrm{SZA}=32^{\circ}$ ) in mid-August 2004. The polarization at $850 \mathrm{~nm}$ (4th panel) contains both $q$ and $u$ values from PMDs 4 (black points) and 7 (blue points), respectively.

this approach would be impractical. Rather, the simulation was optimized for the validation of the SCIAMACHY limb polarization data by assuming

$\langle q\rangle_{\mathrm{PMD}} \equiv q\left(\left\langle\lambda_{\mathrm{PMD}}\right\rangle\right)$,

where the average wavelengths of the PMD measurements $\left\langle\lambda_{\text {PMD }}\right\rangle$ were determined in studies with simulated high- and medium-resolution spectra of the Stokes vector components. These studies showed that there is indeed a good, though tangent-height-dependent, correlation between the polarization at an appropriately chosen wavelength and the effective polarization corresponding to the PMD measurements. The approximate error arising from this approach amounts to less than 0.01 with a small polarization-dependent component that can in principle be corrected for. For PMD 1, where the polarization drops rapidly between about $300 \mathrm{~nm}$ to a minimum around $350 \mathrm{~nm}$ and then slowly recovers, these differences can be mitigated by choosing $\left\langle\lambda_{\mathrm{PMD}}\right\rangle$ to be $375 \mathrm{~nm}$, while for the other PMDs $\left\langle\lambda_{\mathrm{PMD}}\right\rangle$ is about the value noted in Table 1. In order to reduce the sensitivity to absorption features, the simulated wavelength for PMD 5 was set to $1556 \mathrm{~nm}$, just outside the $\mathrm{CO}_{2}$ absorption band. Concerning PMD 5 it should also be noted that emission from the $\mathrm{O}_{2} \Delta$ and $\mathrm{CO}_{2}$ bands cannot be simulated by SCIATRAN. The contribution from emission becomes relevant above $20 \mathrm{~km}$ and dominates the PMD signal above $30 \mathrm{~km}$. As the emission is unpolarized, the measured polarization should therefore be significantly diluted compared to the simulations. For the comparison of the radiances, a few additional wavelengths were selected, taking care that they are outside strong or highly variable absorption.
The TOA radiances were averaged over the vertical extent of the FoV of $0.045^{\circ}$. Atmospheric refraction was not taken into account for this study.

\section{Results}

\subsection{Reflectance and polarization profiles}

Figure 2 shows profiles of the average reflectance $R=$ $\pi I / I_{0}, I_{0}$ being the solar irradiance, at 5 wavelengths (corresponding to the PMD 1 to 5 measurements) and of the average of the retrieved fractional Stokes components $q$ (from PMDs 1 to 5) and $u$ (from PMD 7). The example is for one particular viewing geometry corresponding to one of the reference limb states. The data are from August 2004; the average single scattering angle is $\langle\theta\rangle=92.2^{\circ}$ at a solar zenith angle of about $32.4^{\circ}$. At the single scattering angle around $90^{\circ}$ high average polarization values can be expected. The small solar zenith angle, on the other hand, implies some variability at the longer wavelengths due to the high sensitivity to surface reflectance and tropospheric conditions. The model expectation is plotted as the reddish boxes, their width indicating the variance of the simulated data.

The expected model polarization values and their variance were derived from the model in the following way: a subset of SCIATRAN simulations was selected by requiring $R_{\mathrm{min}}^{\mathrm{data}} \leq R^{\mathrm{SCIATRAN}} \leq R_{\max }^{\mathrm{data}}$ at the closest tangent height step above the climatological tropopause height. For this example, this reference tangent height was at $18 \mathrm{~km}$. The minimum and maximum intensities $R_{\min \text { max }}^{\mathrm{data}}$ were determined for each reference state and each year separately from the 
distribution of the data points, with a margin of $10 \%$ added on both sides to account for possible systematic calibration errors. From the SCIATRAN subset obtained this way, twodimensional distributions of $q$ vs. $R$ and $u$ vs. $R$ were derived (see also Fig. 4 below) for each tangent height. From this two-dimensional histogram a one-dimensional distribution was selected in a narrow slice around the measured reflectance of each individual data point. The expected model polarization value was then estimated as a random value drawn from a Gaussian distribution with the mean and standard deviation of the one-dimensional histogram. The average expected polarization at a given tangent height should then amount to the mean of all model polarization values for all data points, with its variance taking into account both the variance in the reflectance of the data and the intrinsic spread of the model at a given reflectance. This procedure was performed independently for each wavelength and each tangent height, i.e., correlations between wavelengths were not considered.

The modeled average reflectance and its spread were simply derived as the mean reflectance and variance of the SCIATRAN subset at each tangent height. Note that the average reflectance of the model simulations is biased toward high values, although individual scenarios do yield lower reflectances in many cases, ensuring that the data range is well covered. The model distribution was derived for two distinct SCIATRAN setups, where the first, dubbed "set 0", is the basic set described in Appendix B containing only aerosols up to $35 \mathrm{~km}$ and the second, "set 1", has added clouds in the troposphere as well as increased aerosol in the mesosphere. The difference between the simulation sets becomes visible in the average reflectance and polarization at tropospheric and mesospheric tangent heights. In general, the addition of clouds results in slightly higher average radiances and more depolarization at all tangent heights.

Qualitatively, the measured reflectances below about $30 \mathrm{~km}$ behave as expected: at tropospheric heights there is increasing variability with wavelength, while there is less variability at all wavelengths in the stratosphere. Above 30$40 \mathrm{~km}$ the data tend to be significantly higher than the basic (set 0 ) simulations. This behavior is geometry and wavelength dependent and can at least partially (below $50-60 \mathrm{~km}$ ) be explained by the addition of aerosol in the mesosphere, as simulated in set 1.

Concerning the polarization, the obvious discrepancy between model and data at UV-VIS wavelengths is striking. Aside from that, below about $40 \mathrm{~km}$ (25 km for PMD 5), the shape of the profile and the variability of the data seems to be well represented in the model for PMDs 2-5 and 7 . The modeled variance for PMD 1 is too large. This is possibly a consequence of the simulation being only for a single wavelength rather that the average over the complete PMD range with a tangent-height-dependent spectral shape. The zig-zag pattern observed in PMD 1 and 2 is real and originates from the alternating ASM mirror positions at each new tangent height step, which is inherent in the limb scan pattern of SCIAMACHY.

Above $40 \mathrm{~km}$, the SCIATRAN simulations become increasingly unreliable due to the above-mentioned limitations in approximating the spherical geometry (see Sect. 3.1). On the other hand, the data receive a larger contribution from spatial stray light at high altitudes. An assessment of data quality above $\sim 40 \mathrm{~km}$ can therefore only be inconclusive. The apparent offset between simulations and data for PMDs 1 to 3 in the stratosphere, however, cannot be explained by either stray light or model inaccuracies and variance. From the ongoing model intercomparison with Monte Carlo models, it is known that SCIATRAN has a tendency to predict too high depolarization at high altitudes, thus making the difference to the data even more manifest.

\subsection{Variation with viewing geometry}

The fractional Stokes parameters $q$ and $u$ are plotted in Fig. 3 for the same data set (August 2004), this time using all reference states, at a tangent height of $\mathrm{TH} \approx 22 \mathrm{~km}$. The simulated data are derived in the same manner as described above. This plot shows that the observed offsets follow a defined pattern along a typical SCIAMACHY orbit. The results are plotted against the cosine of the single scattering angle that for this particular data set is a unique identifier for each limb profile, but keep in mind that for each single scattering angle there is also a unique solar zenith angle and latitude. For a large range of scattering angles, the polarization values are way too small at wavelengths below $850 \mathrm{~nm}$, and too large above $1500 \mathrm{~nm}$. It should be mentioned that the contribution from emission expected for the PMD 5 measurement would lead to a depolarization rather than too large polarization. Note that the $u$ values for all PMDs but PMD 4 and 7 are derived from the theoretical assumption Eq. (8), i.e., $u=q \cdot u_{\mathrm{SS}} / q_{\mathrm{SS}}$. Since the values for $q$ derived from PMD 1 to 3 are too small or very close to zero, $u$ consequently is too small as well. There are also features at $\cos \theta \approx 0.5$ and -0.8 at which the theoretical assumption completely fails because $q_{\mathrm{SS}} \approx 0$.

It can therefore be concluded that the polarization values as well as the polarization correction in the radiometric calibration of the UV-VIS spectra are highly inaccurate for a large range of wavelengths, scattering geometries and tangent heights.

\subsection{Correlation between polarization and reflectance}

In order to identify the reason for the large discrepancy between model and data, it is useful to look at the correlation between reflectance and polarization at a given wavelength. Starting from a given atmospheric model and assuming sufficiently weak absorption by trace gases or aerosol, increasing the surface reflectance or adding more aerosol should lead to enhanced scattering and therefore increased reflectance. Typically, at tangent heights above the troposphere, the increase 

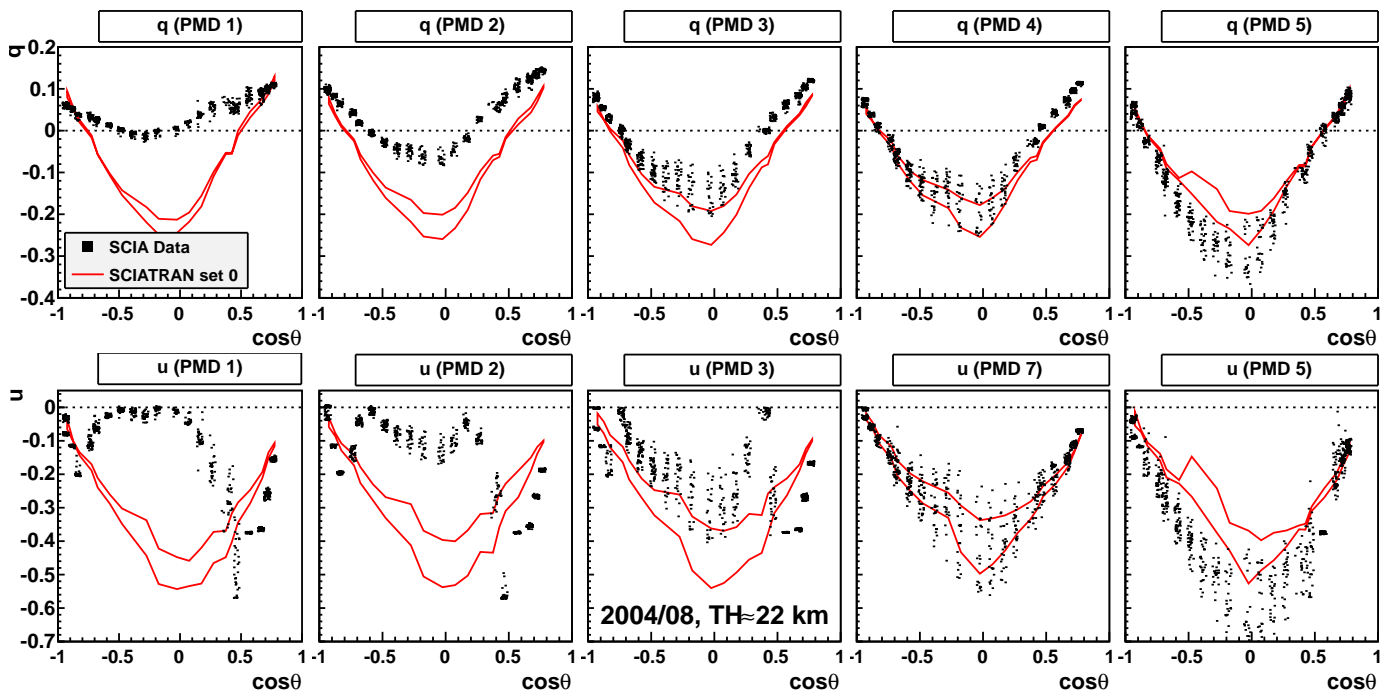

Fig. 3. Values for $q$ and $u$ from the SCIAMACHY data of August 2004 (black points) for each PMD and from SCIATRAN simulations (red lines depicting the $1 \sigma$ envelope of the expected distribution around the mean) at $\mathrm{TH} \approx 22 \mathrm{~km}$.

in reflectance due to the surface or aerosol is much larger than the associated change in the $Q$ and $U$ components of the Stokes vector, resulting in an effective depolarization. Eventually, the values will saturate when the optical thickness along the light path becomes large. The longer the wavelength, the larger the impact of aerosol and surface scattering on both the reflectance and the polarization should be. If the observed discrepancies were due to deficiencies in the modeling of the state of the atmosphere, for instance due to the assumption of Mie scattering on spherical aerosol particles, it should be therefore seen most clearly at the NIR wavelengths.

In Fig. 4, the correlation between $q$ and reflectance $R$ is shown for the same data as in Fig. 2 at a tangent height of about $22 \mathrm{~km}$. The variability in the SCIATRAN data is due to variations of both the stratospheric aerosol load and tropospheric and surface parameters. There seems to be a saturation of the depolarization at high reflectance values. In the SCIAMACHY data, while being well correlated, this relationship does not follow the expected distribution. Even if adding ever more tropospheric or stratospheric aerosol would lead to stronger depolarization in the UV-VIS region, it would do even more so in the NIR region yielding polarization and reflectance values inconsistent with the measurements. It seems unlikely then that the observed differences can be explained by inadequate model simulations.

\subsection{Long-term time dependence}

SCIAMACHY was launched in March 2002 and has ever since experienced a degradation of its scanners and detectors. The throughput loss has been monitored using solar observations along varying instrumental light paths. A large part of it can be explained by the deposition of dirt onto the ASM and ESM mirrors, while a minor part can be attributed to changes in some parts of the optical bench (Snel and Krijger, 2009). To account for the degradation, the concept of $m$ factors (Bramstedt et al., 2009) has been employed in which the measured pixel signals are corrected by a factor $m(t)=I_{\mathrm{S}}(t) / I_{\mathrm{S}}\left(t_{0}\right)$. Here, $I_{\mathrm{S}}$ is the solar radiance or irradiance from the appropriate monitoring measurement at a given time $t$ and the ratio is determined with respect to a reference time $t_{0}$ close to the beginning of stable instrument operations. The $m$ factors correct for a major part of the degradation effects, but they cannot cover for their scan angle and polarization dependence. The relative degradation of the PMDs compared to the science channels is taken care of by the time dependence of the in-band signal that is obtained from the ratio of the virtual sum (cf. Eq. 6) to the PMD signal in measurements of the solar irradiance and that is updated on a daily basis. The monitoring of the polarization sensitivity is not possible with the monitoring measurements, which are all based on unpolarized input.

With the selected data set the time dependence of the polarization values can be investigated directly. Figure 5 shows the evolution of the average reflectance over time for the same reference state as discussed in Sect. 4.1, again at a tangent height of $22 \mathrm{~km}$. The reflectance measurements have been degradation corrected with the $m$ factors. The reflectance data suggest a small relative increase over time that becomes larger at longer wavelengths. Some systematic differences in the mean radiance can be observed between the April and August data, which are most likely due to seasonal differences of atmospheric density and trace gas concentrations in the stratosphere. Conversely, the polarization exhibits a quite clear trend that exceeds the intrinsic variability in the UV-VIS region. All four profiles in this limb state 

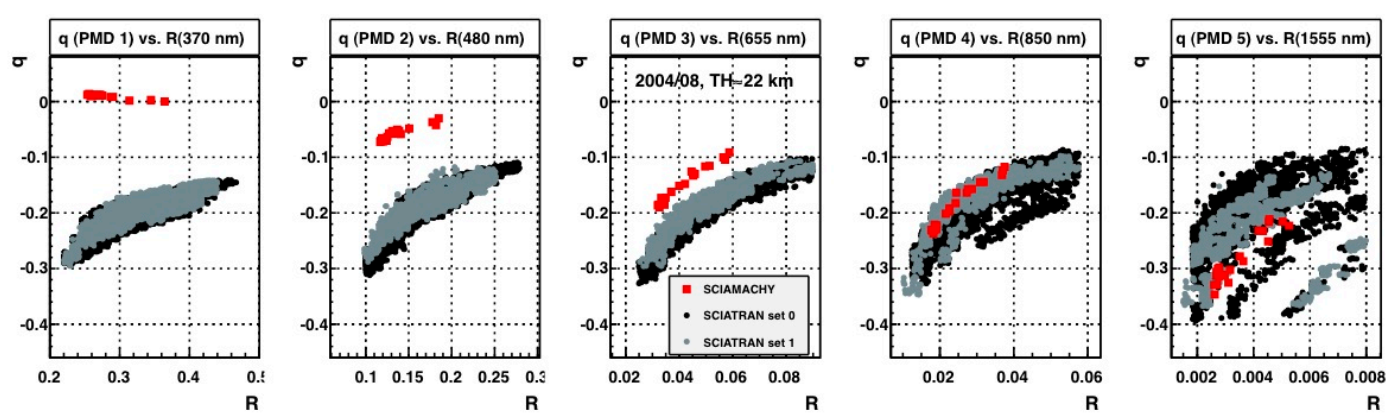

Fig. 4. Correlation between polarization $q$ and reflectance $R$, for the same data as in Fig. 2 (August 2004, reference state 20 , TH $\approx 22 \mathrm{~km}$ ).
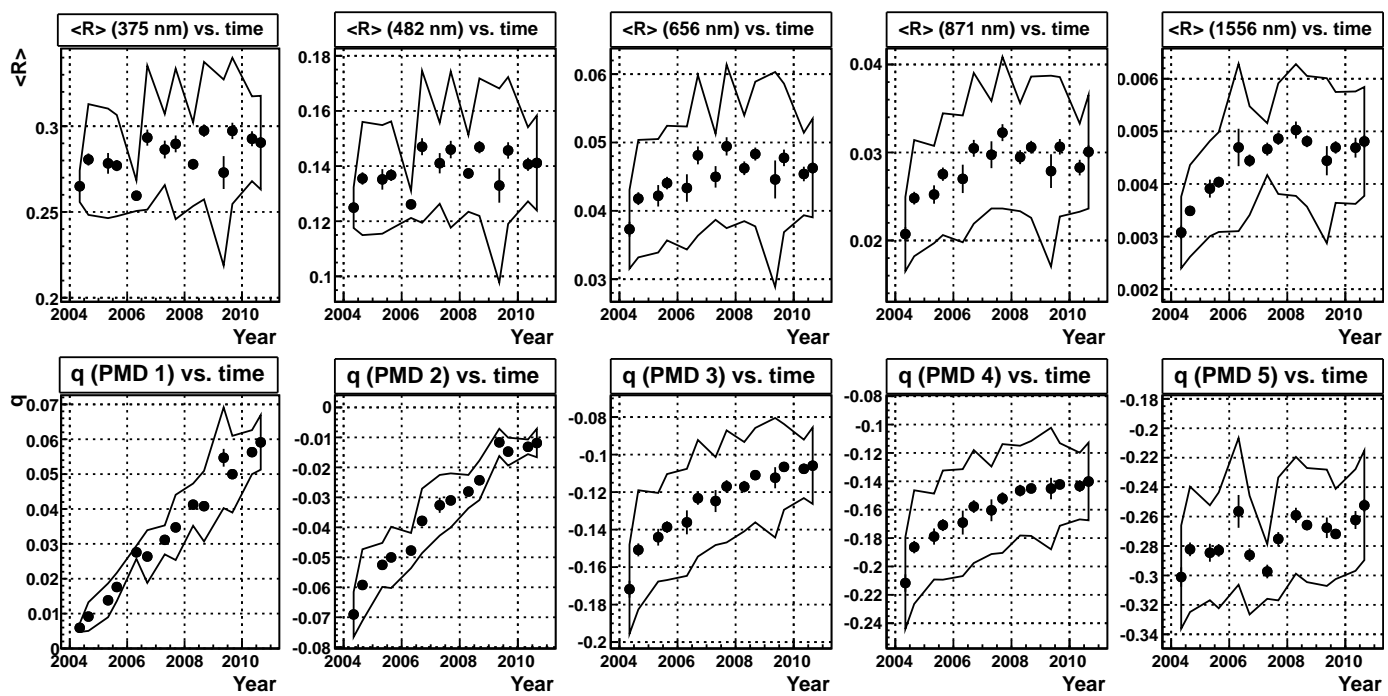

Fig. 5. Time dependence of the average reflectance and polarization. Points with error bars represent the mean and its error, the envelope the variance of the distribution. The data sample corresponds to the reference state 20, the average tangent height is $21.7 \mathrm{~km}$. This plot contains both the April and August data samples.

exhibit a similar behavior, thus not revealing any significant scan angle dependence. Comparing with Fig. 4, the trends in the polarization are consistent with the trends in the reflectance only for PMDs 4 and 5; for PMDs 1 to 3 they seem to be too large. That means that the observed trends cannot be explained with the simulated relationship between the mean reflectance and mean polarization alone. Of course, provided that the reflectance increase is really due to changes in atmospheric composition (albedo, cloud cover, aerosol), it cannot positively be excluded that in the UV-VIS region these changes "conspire" in way to generate trends in the polarization along the vertical axis of Fig. 4. It is more likely that instrumental changes inside the OBM affecting the polarization sensitivity can cause the observed depolarization trends. Eventually, this ambiguity can only be solved by a rigorous analysis involving a retrieval of albedo and aerosol composition combining all wavelengths and using more realistic model simulations. However, prior to that the large offset in the polarization values between model and data that have ap- peared already at the beginning of life of the instrument has to be understood.

\section{Error sources and in-flight recalibration}

\subsection{Assessment of error sources}

The large spread in the model predictions, the uncertainties in the parameterization of the state of the atmosphere and the intrinsic model errors of $\mathcal{O}(0.01)$ cannot explain the observed differences to the measured data. The remaining two other options are a failure of the polarization algorithm and errors in the calibration key data.

The initial inputs to the polarization algorithm are the PMD and the science detector signals weighted with the ratio of their throughputs and integrated over the PMD spectral range. Equation (6) can be rewritten as

$\operatorname{IB} \frac{S^{\mathrm{P}}}{V_{0}}=\left\langle\frac{1+\mu_{2}^{\mathrm{P}} q+\mu_{3}^{\mathrm{P}} u}{1+\mu_{2}^{\mathrm{D}} q+\mu_{3}^{\mathrm{D}} u}\right\rangle \quad$ with 
$V_{0}=\sum_{i} S_{i}^{\mathrm{D}} M_{1, i}^{\mathrm{PD}}$

In the algorithm, $q$ and $u$ are assumed to be constant. Also, the numerator in the average term of Eq. (11) is usually dominating over the denominator, such that it is possible to approximate

$\left\langle\frac{1+\mu_{2}^{\mathrm{P}} q+\mu_{3}^{\mathrm{P}} u}{1+\mu_{2}^{\mathrm{D}} q+\mu_{3}^{\mathrm{D}} u}\right\rangle \approx \frac{1+\left\langle\mu_{2}^{\mathrm{P}}\right\rangle q+\left\langle\mu_{3}^{\mathrm{P}}\right\rangle u}{1+\left\langle\mu_{2}^{\mathrm{D}}\right\rangle q+\left\langle\mu_{3}^{\mathrm{D}}\right\rangle u}$

without changing the results noticeably. The averages of the MMEs, $\mu_{2,3}$, can be determined for each measurement as

$\left\langle\mu_{n}^{\mathrm{P}, \mathrm{D}}\right\rangle=\frac{1}{V_{0}} \sum_{i} S_{i}^{\mathrm{D}} M_{1, i}^{\mathrm{PD}} \mu_{n i}^{\mathrm{P}, \mathrm{D}}, \quad n=2,3$.

In particular, for the case of PMD 1 the denominator in Eq. (12) is very close to 1 within $1 \%$ due to cancelations of positive and negative values of $\mu_{2,3}^{\mathrm{D}}$, implying that

$\mathrm{IB} \frac{S^{\mathrm{P}}}{V_{0}}-1 \approx\left\langle\mu_{2}^{\mathrm{P}}\right\rangle q+\left\langle\mu_{3}^{\mathrm{P}}\right\rangle u$.

The black points in the bottom panel of Fig. 6 show the lefthand side (LHS) of this equation for PMD 1 as a function of the single scattering angle $\cos \theta$. Likewise, the red curve in this figure shows the expectation for the right-hand side (RHS) of Eq. (14) determined from the expectation values of SCIATRAN for $q$ and $u$ (magenta and green curves in the top panel) using the average $\mu_{i}$ according to Eq. (13) from the on-ground polarization key data. Clearly, neither the shapes nor the magnitude of the curves match.

The influence of each of the three calibration terms can now be examined separately. The in-band signal IB is essentially a scale factor for the relative calibration of PMD and science detectors. A change in the in-band signal by a factor $f$ close enough to 1 would first-order shift the entire curve by $1-f$. On the other hand, a change in the major MME, $\mu_{2}^{\mathrm{P}}$, would mainly scale the curve, while a change in in the minor MME $\mu_{3}^{\mathrm{P}}$ would alter the shape of the curve proportional to the shape of $u$. Assuming that the model values for $q$ and $u$ resemble the true atmospheric polarization in a reasonable fashion, this figure shows that the sensitivities to $q$ and $u$ of the PMD signal cannot be described with the average $\mu_{2,3}$ determined from the on-ground key data. Reversely, it should be possible to "tune" the respective values in the SCIATRAN curve until it matches the data. This has been done for the blue curve in the bottom panel of Fig. 6 by changing $\left\langle\mu_{2}^{\mathrm{P}}\right\rangle$ to 0.8 and $\left\langle\mu_{3}^{\mathrm{P}}\right\rangle$ to -0.45 from their original values of 0.98 and -0.11 , respectively. The remaining small discrepancies may be due to a shift in the in-band signal, the contribution of the polarization to the integrated science detector signal and the above-mentioned intrinsic model errors.

From the above discussion it seems evident that the onground key data do not resemble the in-flight polarization sensitivities. A drastic change such as that observed in the
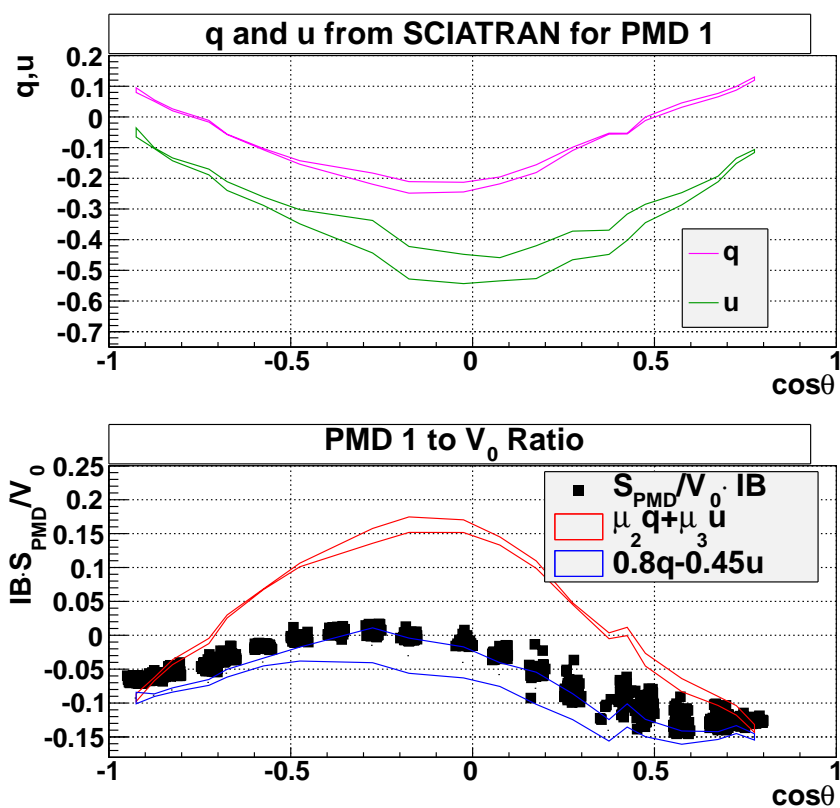

Fig. 6. Top panel: expectation values for $q$ and $u$ for August 2004 data at $\mathrm{TH} \approx 21.7 \mathrm{~km}$ from SCIATRAN set 0 ; the curves depict the RMS around the mean value. Bottom panel: the points are the data for PMD 1 (LHS of Eq. 14) and the red curve represents the RMS around the expectation value of the RHS of this equation using averages of on-ground key data, while the blue curve represents the values obtained by setting $\left\langle\mu_{2}^{\mathrm{P}}\right\rangle=0.8$ and $\left\langle\mu_{3}^{\mathrm{P}}\right\rangle=-0.4$.

UV close to the beginning of life of the instrument can also not be explained by the rather gradual scanner degradation. A likely reason for the observed behavior is a phase shift within the optical bench of the instrument. A temperaturedependent phase shift in the predisperser prism that splits the beam and directs the light onto the individual detector channels and the PMDs had been observed already during the on-ground calibration measurements and is the main reason for the initial PMD sensitivity to $45^{\circ}$ polarized light (Snel, 1999). This initial $u$ dependence is reflected in the $\mu_{3}^{\mathrm{P}}$, which is largest at UV wavelengths and then smoothly drops off. It is quite conceivable then that stress birefringence induced mechanically in flight by the lack of gravity or by temperature gradients within the instrument could have altered the initial polarization phase shift.

Other, minor causes may be the use of the in-band signal as determined from solar measurements with the ESM diffuser where the spectrally weighted average of the virtual sum might be different than for Earth shine spectra. There are indeed some systematic differences between the solar measurements and the in-band signal as determined from nadir measurements where $\theta \approx 180^{\circ}$. In addition, even for zero input polarization, the in-band signal can be affected by the phase shift as well. An additional minor, though nonnegligible effect may be due to the above-mentioned ambiguities in the polarization frame definition for the on-ground 
calibration measurements. A recent reanalysis using independent calibration data shows that the signs of some minor MMEs (e.g., $\mu_{3}^{\mathrm{D}}$ ) may be wrong in some cases (Krijger et al., 2009).

The unexpectedly small PMD-to-detector signal ratios also explain the failure of the polarization algorithm, as seen in Fig. 3. Using $u / q=$ const. (Eq. 8), Eq. (14) can be solved for $q$ :

$q \approx \frac{1}{\mu_{2}^{\mathrm{P}}}\left(\mathrm{IB} \frac{S^{\mathrm{P}}}{V S_{0}}-1\right) /\left(1+\frac{\mu_{3}^{\mathrm{P}}}{\mu_{2}^{\mathrm{P}}} \frac{u_{\mathrm{SS}}}{q_{\mathrm{SS}}}\right)$.

A small value of the numerator would now automatically imply a small value of $q$, and therefore a small value of $u$. In the original Eq. (14), however, small values of the left-hand side can just as well be explained by a partial cancelation of both terms on the right-hand side. Without an absolute input value for $u$, the virtual sum equation will always yield a small $q$ if the contributions of $\mu_{2}^{\mathrm{P}} q$ and $\mu_{3}^{\mathrm{P}} u$ are opposite and comparable, as is the case for most of the limb-viewing geometries of SCIAMACHY.

\subsection{Recalibration of polarization sensitivity using in-flight data}

The approach taken in the previous section to explain the measured PMD signals can be reversed to find the values for the MMEs that yield polarization values compliant with the expected ones from the model. Here, a method is presented that is to be considered a first step towards a recalibration of the polarization key data, thus providing information on the sensitivity of the data to these parameters and allowing to identify and address potential issues.

The same data set as described in Sect. 2.3 is used, and the matching SCIATRAN sets have been selected according to the method described in Sect. 4.1. That means, for each of the 14 independent data sets (one for April and August each year between 2004 and 2010), a corresponding SCIATRAN subset with the modeled intensities lying in the same range as the data at a given reference height was selected. From each such subset, histograms as shown in Fig. 4 were generated, separately for all of set 0 and set 1 (see Sect. 4.1 and Appendix B), as well as for two subsamples of set 0 containing either stratospheric background or volcanic aerosol.

Similarly to the procedure explained in Sect. 4.1, a mean value $q_{\mathrm{S}}$ is determined for the model, and the difference between $q_{\text {Data }}$ and the model expectation value $q_{\mathrm{S}}$ is minimized by adjusting the three fit parameters IB, $\mu_{2}^{\mathrm{P}}$ and $\mu_{3}^{\mathrm{P}}$. Details of the fit methods are explained in Appendix C. The fit was carried out for one tangent height step above the reference tangent height. The values for the MMEs obtained with this method thus correspond to the ones that make the data agree on average with the mean value of the model given the reflectance for each measurement point. The advantage of this method compared to fitting the data on an individual profile basis is that at least some model uncertainties arising from inappropriate parameter settings and profile shapes can be mitigated. A profile-by-profile fit would also require an iterative adjustment of the model similar to optimal estimation retrievals, which would be forbiddingly slow.

\subsection{Preliminary results}

Figure 7 shows the results for the three fit parameters. The fitted in-band signal is plotted in the top row. The colored curves show the results for the individual SCIATRAN subsamples as described above; the black points with the gray shaded error band give, for each point in time, the result for the SCIATRAN sample with the lowest $\chi^{2}$ and its error. This curve is shown only to give an impression on the associated intrinsic fit errors, not to make a judgment on the goodness of fit with respect to each subset. The dashed line shows the in-band signal derived from the solar measurements that is used in the operational Level 0-1 processor. The trend in this in-band signal is well captured by the fit. The observed offsets, which are significant for all but PMDs 4 and 7, are by and large consistent with an independent analysis of nadir backscattering data at $\theta \sim 180^{\circ}$. There is also some dependence on the chosen model subset that will have to be regarded as a systematic fit error.

In the middle row the sensitivity to the major polarization component of the respective PMDs (i.e., $\mu_{2}^{\mathrm{P}}$ for PMDs 1-5 and $\mu_{3}^{\mathrm{P}}$ for PMD 7) is shown, with the same coding for the individual curves. The dashed lines here indicate the mean values of the corresponding on-ground calibration key data. The intrinsic fit error on this MME is much larger, but it is still obvious that it differs significantly from the on-ground calibration data for PMD 1 and PMD 2, and exhibits a significant trend there. The difference between simulation sets is smaller than the fit parameter uncertainty, although the values obtained from set 1 are systematically lower for PMD 1 and 2.

The fit results for $\mu_{3}^{\mathrm{P}}$ (PMD 1-5) and $\mu_{2}^{\mathrm{P}}$ (PMD 7) are shown in the bottom row, with the perhaps most surprising result that the sensitivities to $u$ in PMDs 1-3 are much larger than the initial on-ground measurements suggest. The model dependence is relatively large and increases with wavelength. A significant trend can be observed for PMDs 1 to 3 as well. As already discussed above, with this method it is not possible to unambiguously decide whether these trends are caused by actual trends in the physical state parameters that are not captured by the model or by instrumental change. From the fact that the behavior of the fitted in-band signal agrees well with model-independent measurements, it can be concluded, though, that the trends are at least partially due to instrument degradation.

The main difference between set 1 and set 0 are tropospheric clouds in set 1 giving rise to depolarization (compared to cloudless scenes) that is larger at longer wavelengths. In general the fit parameters, in particular $\mu_{2}^{\mathrm{P}}$ and $\mu_{3}^{\mathrm{P}}$, are highly correlated. This means that there is an ambiguity 


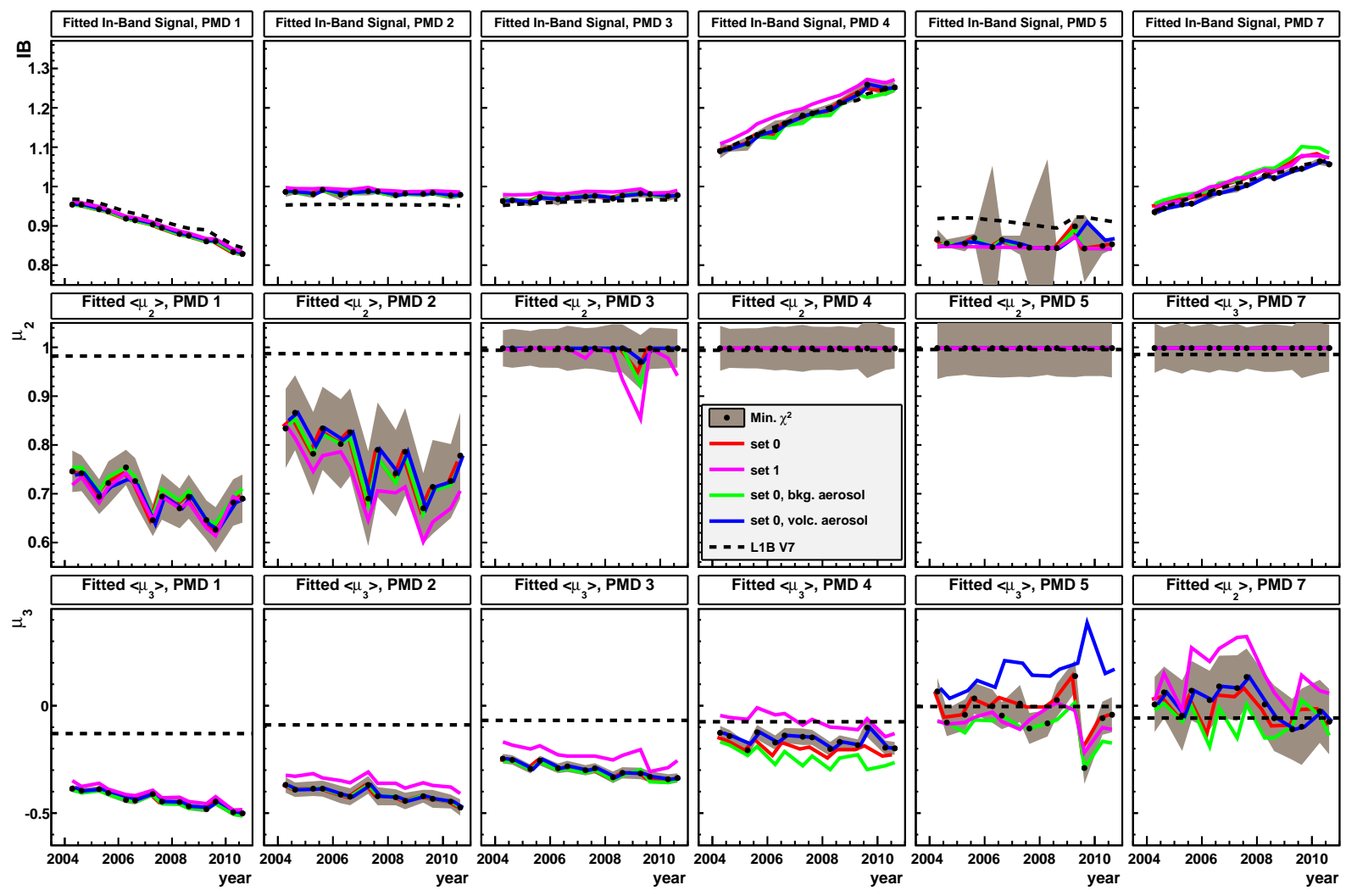

Fig. 7. Results of the key data fits to SCIATRAN vs. time. Top panels: in-band signal for PMDs $1-5$ and 7 , middle panels: $\left\langle\mu_{2}^{\mathrm{P}}\right\rangle$ for PMDs 1-5 and $\left\langle\mu_{3}^{\mathrm{P}}\right\rangle$ for PMD 7, bottom panels: $\left\langle\mu_{3}^{\mathrm{P}}\right\rangle$ for PMDs 1-5 and $\left\langle\mu_{2}^{\mathrm{P}}\right\rangle$ for PMD 7. The black points with the fill area correspond to the values for best fitting SCIATRAN subset for each point in time and their errors. The colored curves are the results for the individual SCIATRAN samples and the dashed curve shows the values that are actually used in the Level 0-1 algorithm.

in the fit between genuine depolarization and cancelation of $\mu_{2}^{\mathrm{P}} q$ and $\mu_{3}^{\mathrm{P}} u$ in the PMD signal. It is therefore likely that, for the cloudless scenes in set 0 , the fit compensates the depolarizing effects of actual clouds by increasing the magnitude of $\mu_{3}^{P}$ - and thereby decreasing the PMD signal. In addition to the obvious systematic differences between the results for set 0 and set 1 , there may also be a small seasonal component in the fit parameters for PMDs 1 to 3, which could be related to a seasonal variation of cloud cover. Note, though, that the effect of clouds is overestimated by SCIATRAN, both due to the assumption of homogeneous layers and because of the aforementioned intrinsic model errors. The set 1 simulations are therefore not necessarily more realistic.

The fit residuals are wavelength and viewing geometry dependent, with the maximum values reaching from about 0.01 for PMD 1 to a few $10^{-2}$ for PMDs 2 to 4 to around 0.1 for PMD 5 and 7. This is about the expected range when regarding both model and measurement errors.

\subsection{Discussion}

The results presented here show that it is in principle possible to recalibrate the polarization sensitivities using a model for the expected polarization and in-flight data. In particular, the good representation of the in-band signal by the fit compared to the solar reference measurements as well as to nadir measurements (not shown here) indicate that even this very reduced data set used here has sufficient sensitivity to extract information about the time-dependent behavior of the MMEs. The method can of course later be expanded to a more extended data set covering other seasons and more geometries, yielding more independent combinations of $q$ and $u$ and thereby reducing correlations between the resulting fit parameters. It can also be refined with respect to all aspects of the model simulations. It is obvious that systematic errors in the model will lead to systematic errors in the derived MMEs. For further investigations it is important that the model dependence be understood and reduced. Still, even if the MMEs can only be constrained to a few $10^{-2}$ within model uncertainties, the polarization correction to the radiometric calibration can be considerably improved. It remains to be seen, though, to what extent the physical information content regarding stratospheric aerosols and the like in the polarization data itself will be impacted by inevitably linking it to a model for their calibration. 
The observed behavior of the MMEs, in particular the wavelength dependence of $\mu_{3}^{\mathrm{P}}$, is indeed indicative of a polarization phase shift generated by the predisperser prism (Snel, 1999; Frerick, 1999). Important information about its magnitude and time dependence can be gained from these fit results. Likewise, it should be possible to directly fit the phase shift rather than individual end-to-end MMEs by means of an instrument model as described in Snel and Krijger (2009) by adding a retarding element to it.

The results shown and discussed here are therefore to be considered a preliminary but useful step towards an in-flight recalibration of the polarization sensitivity of SCIAMACHY. They can as well serve the purpose of discussing the implications for the polarization measurements and the polarization correction:

- First, the observed enhancement of the sensitivity to $u$ in the UV-VIS brings about an enhancement of the complications due to the polarization algorithm discussed in Sect. 5.1 (Eq. 15). That means, even if the true polarization sensitivities were known with high accuracy, the current algorithm would still fail to give large polarizations for both $q$ and $u$. The algorithm needs to be changed in order to provide a fixed estimate for $u$ directly to the virtual sum equation. The estimate can be based on assumptions such as Eq. (8), or, more appropriate for the limb mode where the variability in this ratio is large, a model estimate from the relationship between $u$ and the measured reflectance. The large sensitivity to $u$ implies that the accuracy of $q$ will be severely impacted by the uncertainty in the estimate for $u$.

- Another important issue to note is that the fit does not actually deliver the polarization sensitivities $\mu_{2,3}^{\mathrm{P}}$ of the PMDs but rather an effective combination of PMD and detector sensitivities ${ }^{4}$. From the fit alone, it is not possible to derive the detector sensitivities separately. Without an instrument model that can describe the observed changes in the effective polarization sensitivities, it is therefore not possible to derive the detector sensitivities needed for the polarization correction to the radiometric calibration. In particular, the effect on the nadir polarization measurements and calibration remains unclear. If the cause for the changes is a phase shift in the predisperser prism, such an instrument model would be available and could in principle be used to infer the phase shift directly from the fits. The Mueller matrix for each relevant light path and for both science detectors and PMDs can then be derived from the model (Snel and Krijger, 2009).

\footnotetext{
${ }^{4}$ Note that the correction for the initial values of the detector MME is contained in the factor $c_{\mathrm{d}}$ in Eq. (C1). A systematic error in these MMEs would therefore result in a systematic error on the fitted MMEs.
}

\section{Conclusions}

A rigorous study has been performed to assess the quality of the SCIAMACHY limb polarization measurements. Comparison with SCIATRAN simulations revealed large discrepancies between model and data that are most prominent in the $\mathrm{UV}$ and visible regions. These discrepancies are outside the range of possible model uncertainties. In the UV, differences between measured and predicted polarization values amount to as much as 0.25 for $q$ and 0.5 for $u$. There is a clearly systematic behavior with the viewing geometry along a typical orbit. The discrepancies can be ultimately related to an instrumental change of the polarization sensitivities and, in addition, to a subsequent failure of the polarization algorithm to determine the correct polarization values. Erroneous or ineffective polarization corrections lead to errors in the absolute radiometric calibration of up to $15 \%$. Also, the spectral shape of the measured radiance may be impacted - for instance in the region around $350 \mathrm{~nm}$, where the polarization sensitivity has some particular spectral features. The discussion here concentrated on the limb data; however, nadir data may be affected as well, albeit to a lesser extent.

The model can be used to recalibrate the effective polarization sensitivity of the instrument with in-flight limb data. Preliminary results indicate that it is possible to derive the relevant parameters from fits of the data to the model. The fit results reveal a dramatic shift of the in-flight polarization sensitivities compared to on-ground calibration measurements, hinting at a phase shift inside the instrument's optical bench module as the likely cause. The accuracy of the fit needs to be further improved by extending the number of independent data points and reducing the sensitivity to model uncertainties. Alternative methods are currently being investigated.

Eventually, the results of this study and further investigations will lead to an improved understanding of the instrument behavior and possibly a recalibration of the (time-dependent) polarization sensitivities. By adapting the polarization algorithm properly, the accuracy of the polarization data can be considerably improved.

\section{Appendix A}

\section{Polarization algorithm details}

The polarization algorithm uses Eq. (6). The pixel signals $S_{i}$ are derived from the raw ADC counts delivered in the Level 1b (L1B) product (version 7.03/7.04) and corrected for additive offsets and the limb dark signal in the same manner as described in Slijkhuis (2008). The limb dark signal correction is performed by subtracting the signal measured during the last scan of each limb state at an altitude of $250 \mathrm{~km}$. The in-band signal is taken from the auxiliary Sun Mean Reference file, which contains calibrated measurement results from the solar reference measurement of the same day. The 
key data used to calculate the MMEs are identical to the ones used in the operational processor. The MMEs are interpolated to the scan angles encountered in each measurement. $\mathrm{Bad}$ or dead detector pixels specified in a bad and dead pixel mask (BDPM) delivered with the L1B product are excluded from the virtual sum, and their values are replaced by an interpolation involving the adjacent good pixels.

The virtual sum equation is solved using the Brent rootfinding algorithm. For PMDs 1-5 the assumption Eq. (8) is made for $u$ if $|q|>0.02$. If $|q|<0.02, u=c u_{\mathrm{SS}}$ with $c \approx 0.8$. The value of $u$ is recalculated in each step of the iteration. If in an iteration step $q^{2}+u^{2}>q_{\mathrm{SS}}^{2}+u_{\mathrm{SS}}^{2}, u$ is adjusted to $u= \pm \sqrt{q_{\mathrm{SS}}^{2}+u_{\mathrm{SS}}^{2}-q^{2}}$, with the sign fixed by the sign of $u_{\mathrm{SS}}$. This strategy to adjust $u$ causes the virtual sum to have more than one solution in some cases in the nadir mode, from which only one (not necessarily the correct one) will be identified. There is no indication that this happens with limb data as well.

Below the differences of the algorithm used in this study and the operational L1B processor are listed.

- The polarization algorithm was applied to all tangent heights. In the operational algorithm, above $30 \mathrm{~km}$ an extrapolation using the single Rayleigh scattering value is used.

- No memory effect correction was applied to the pixel detector signals. The memory effect is a residual signal from the previous exposure. During a step from one tangent height to the next in a limb scan, a pixel exposure is taken but not stored in the data, with the consequence that for the first exposure after each step no information on the previous signal is available. Instead, an interpolation between the signals at the two tangent heights is made. Due to a bug in the interpolation routine of the processor, the memory effect was wrongly calculated for each first readout in a limb scan, leading to visible artifacts both in the limb radiance and the polarization above approx. $40 \mathrm{~km}$. As the memory effect is very small between 20 and $30 \mathrm{~km}$ and hardly changes anymore above $30 \mathrm{~km}$, by omitting the memory effect correction these artifacts can be significantly reduced, while retaining radiometric accuracy.

- The PMD signals were filtered for spikes caused by energetic particles hitting the detectors. The frequency of these spikes is not very high outside the SAA region, but if they occur they can cause significant polarization errors above about $40 \mathrm{~km}$. PMD 7 is the one affected most by spikes, followed by PMD 1 . The efficiency of the filtering algorithm is not $100 \%$ so that quite generally above $55 \mathrm{~km}$, the PMD 7 values can be considered unreliable.

- Similarly to the PMDs, also the science detector pixels can be hit by particles. In the operational processor a check was implemented to identify hot pixels during the measurement of the limb dark signal at $250 \mathrm{~km}$ tangent height. This check was not implemented here, resulting in a slightly different limb dark signal subtraction. Outside the SAA, hot pixels occur only rarely such that the impact on the virtual sum is minuscule.

- As mentioned above, bad pixels specified in the BDPM are not used in the virtual sum. In channel 6 above $1585 \mathrm{~nm}$ a significant number of pixels suffers from a so-called random telegraph signal (RTS), which causes their dark currents to randomly jump between two or more different values. These pixels are not necessarily flagged as bad in the BDPM; however, they can significantly spoil the virtual sum when the overall radiance is low. Pixels subject to RTS were identified from the distribution of signals in the limb dark measurement at $250 \mathrm{~km}$ and their signal replaced by an interpolation in wavelength. Based on data from 2010, there are only approx. 40 good, stable pixels left in the PMD 5 spectral region above $1585 \mathrm{~nm}$. The interpolation therefore relies on the signal shape being smooth over most of the relevant spectral range. Model studies indicate that this is the case for tangent heights above the tropopause. Above $20 \mathrm{~km}$, instead of an interpolation parameters of a fit of the pixel signal normalized to the solar reference signal $S_{0}$ of the form $\log \left(S / S_{0}(\lambda)\right)=p_{0}+p_{1}\left(\lambda-\lambda_{0}\right)$ is applied. Still, the results for the polarization obtained from PMD 5 above about $25 \mathrm{~km}$ have to be considered unreliable.

- An additional requirement on the pixel signal-to-noise ratio to be above -3.5 is imposed, again rejecting pixels whose dark currents or electronic offsets may be overestimated.

- The polarization value from PMD 7 (essentially $u$ ) is not calculated in the operational processor. Here, it is calculated by iterating the $q$ value obtained from the PMD 4 measurement with the $u$ value obtained from PMD 7 . PMDs 4 and 7 cover the same wavelength range; it is therefore possible to get an independent value of $q$ and $u$ for $\langle\lambda\rangle \approx 850 \mathrm{~nm}$. As mentioned in Sect. 2.2, the value of $\mu_{3} u$ is inconsistent with that entering the virtual sum equations of the other PMDs; therefore, the value of $\mu_{3}^{\mathrm{P}}$ for PMD 7 has been replaced by $-\mu_{3}^{\mathrm{P}}$.

\section{Appendix B}

\section{SCIATRAN scenario details}

\section{B1 Aerosol extinction profiles}

For the basic SCIATRAN simulations, 4 aerosol layers were defined: 


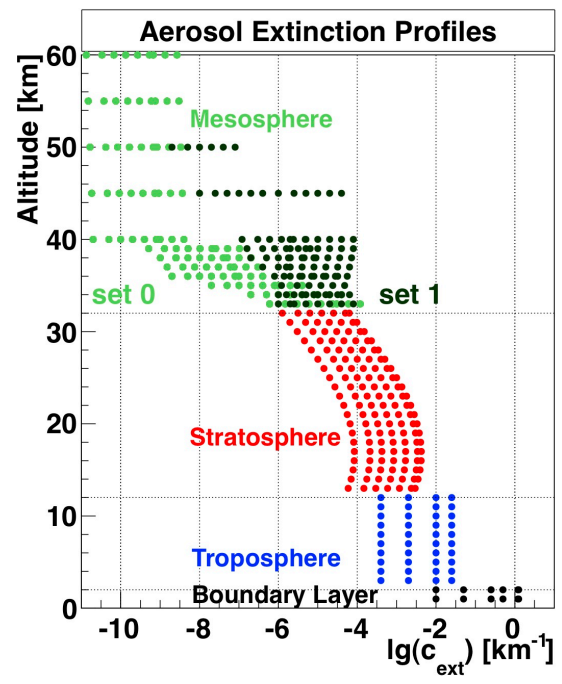

Fig. B1. Aerosols extinction coefficient profiles used in the SCIATRAN simulations. The volume extinction coefficient is given at $550 \mathrm{~nm}$.

- The boundary layer reaching from the surface to $2 \mathrm{~km}$. It can consist of either urban or maritime aerosol, distributed uniformly between 0 and $2 \mathrm{~km}$.

- The tropospheric layer consisting of continental aerosol distributed uniformly between 3 and $12 \mathrm{~km}$.

- The stratospheric layer consisting of either background or volcanic aerosol distributed roughly according to the ECSTRA model (Bingen and Fussen, 2000) for background conditions. The difference between the background stratospheric and volcanic aerosol types is mainly that a small fraction of coarse volcanic ash particles has been added to the sulphuric acid droplets typically contained in the background stratospheric aerosol.

- A mesospheric layer between 32 and $100 \mathrm{~km}$. In the standard simulation set 0 , the mesospheric aerosol is of the same type as the stratospheric aerosol, and the profile above $35 \mathrm{~km}$ drops rapidly to a volume extinction coefficient below $10^{-8} \mathrm{~km}^{-1}$ (light-green points in Fig. B1), which is below the sensitivity of the data. For the simulation set 1 , the density of the mesospheric aerosol is increased (dark-green points in Fig. B1) and its type is varied among the aerosol types in Table B1. Currently, there is no specific model for mesospheric aerosol (e.g., meteoric dust) implemented in SCIATRAN. The main purpose of these simulations was to investigate if at all any additional aerosol at high altitudes could explain the scattering angle and wavelength-dependent excess radiance observed in the data.
Table B1. Aerosol layers used in SCIATRAN scenarios. BL, TL, SL and ML stand for boundary, tropospheric, stratospheric and mesospheric layers. The aerosol types are the maritime (M), continental (C), urban (U), stratospheric background (B) and volcanic aerosol type defined in the WMO report (Deepak and Gerber, 1983; Bolle, 1986).

\begin{tabular}{lrll}
\hline Layer & Top height & Type & AOD \\
\hline BL & 2 & M,U & $0.02-2.5$ \\
TL & 12 & C & $0.004-0.25$ \\
SL & 32 & B,V & $0.0008-0.08$ \\
ML & 100 & B,V,U,C & $10^{-6}-10^{-4}$ \\
\hline
\end{tabular}

Table B1 summarizes the aerosol types and loads for the main SCIATRAN simulation set. Figure B1 shows the extinction profiles (at $550 \mathrm{~nm}$ ). Any aerosol profile and type in a layer can be combined with any other profile and type in another layer. Ultimately, about 900 different aerosol scenarios have been simulated.

\section{B2 Studies on profile variations}

The influence of variations in temperature, pressure and $\mathrm{H}_{2} \mathrm{O}, \mathrm{O}_{3}$ and $\mathrm{NO}_{2}$ concentrations on the radiances and polarization values was investigated in a separate study. Pressure and temperature variations lead to variations in the radiance of up to $15 \%$, with the largest impact at $850 \mathrm{~nm}$ and at large scattering angles. Contrary to that, the polarization varied by less than 0.008 and 0.015 for $q$ and $u$, respectively, with the maximum again around $850 \mathrm{~nm}$. The water vapor column was varied between 0.01 and $8 \mathrm{~g} \mathrm{~cm}^{-2}$, the resulting maximum deviations were $15 \%$ in the radiance, 0.02 in $q$ and 0.03 in $u$ at $650 \mathrm{~nm}$. Ozone columns were varied between 100 and $500 \mathrm{DU}$, resulting in up to $20 \%$ variations in intensity, but less than a few $10^{-3}$ in polarization between 450 and $700 \mathrm{~nm}$. The maximum impact of $\mathrm{NO}_{2}$ variations amounted to a few percent in radiance and less than 0.002 in the polarization.

\section{Appendix C}

\section{Minimization procedure for the fit of the key data to the model}

The fit procedure scans the fit parameters IB, $\mu_{2}^{\mathrm{P}}$ and $\mu_{3}^{\mathrm{P}}$. The fit result is obtained for the point in this three dimensional parameter space, for which

$$
\chi^{2}=\sum_{i} \frac{\left(\frac{1}{\left\langle\mu_{2}^{\mathrm{P}}\right\rangle}\left\{\mathrm{IB}\left[\frac{S^{\mathrm{P}}}{V_{0}}\right]_{i} c_{d}-1-\left\langle\mu_{3}^{\mathrm{P}}\right\rangle\left\langle u^{\mathrm{S}}\right\rangle_{i}\right\}-\left\langle q^{\mathrm{S}}\right\rangle_{i}\right)^{2}}{\left(\mu_{3}^{\mathrm{P}} \delta u_{i}^{\mathrm{S}}\right)^{2}+\left(\delta q_{i}^{\mathrm{S}}\right)^{2}}(\mathrm{C} 1)
$$


reaches its minimum value ${ }^{5}$. The factor

$c_{\mathrm{d}}=1+\left\langle\mu_{2}^{\mathrm{D}}\right\rangle\left\langle q^{\mathrm{S}}\right\rangle_{i}+\left\langle\mu_{3}^{\mathrm{D}}\right\rangle\left\langle u^{\mathrm{S}}\right\rangle_{i}$

uses the on-ground calibration key data as an initial estimate to take into account the polarization effect on the science detector signals. The expected values $\left\langle q^{\mathrm{S}}\right\rangle_{i}$ and $\left\langle u^{\mathrm{S}}\right\rangle_{i}$ are the mean values of the model in a small one-dimensional slice of the two-dimensional $q$ vs. $R$ model histogram (see Fig. 4) around the reflectance value of each data point $i$. The model errors are the squared sums of the variances of the values in this one-dimensional slice and an ad hoc constant systematic error assigned to each wavelength based on the discussions in Sect. 3.1 and Appendix B, which amounts to about 0.01 for PMD 1 and increases to about 0.03 for PMD 5.

Technically, the fit is performed in steps of IB scanning the 2-dimensional $\left(\mu_{2}^{\mathrm{P}}, \mu_{3}^{\mathrm{P}}\right)$ surface. The resulting $\chi^{2}$ distribution does not correspond to that of a normal distribution as the true errors are unknown. An estimate for the errors of the fit parameters is therefore obtained by normalizing the $\chi^{2}$ distribution such that $\chi_{\min }^{2} / \mathrm{ndf}=1$, where ndf is the number of degrees of freedom, i.e., the number of data points used in the fit, and then calculating the probability distribution for each point $\left(\mu_{2}^{\mathrm{P}}, \mu_{3}^{\mathrm{P}}\right)$. From this distribution, the correlation matrix is determined using the (co)variances along each direction. The error on IB is treated independently from that, neglecting the possible correlations between IB and $\mu_{2,3}$. The $\chi^{2}$ vs. IB function is fitted by a second-order polynomial that is then used to estimate both the minimum values and the values at which $\chi^{2}=\chi_{\min }^{2}+1$, which serve as an estimate for the fit uncertainty. Obviously, this method serves only as a rough estimate of the fit errors and needs to be refined eventually.

Acknowledgements. SCIAMACHY is a national contribution to the ESA ENVISAT project, funded by Germany, The Netherlands, and Belgium. SCIAMACHY Level 1 data have been provided by the ESA. We thank the SCIAMACHY Quality Working Group team and especially R. Snel and J. M. Krijger at SRON for the continuing support and helpful discussions. We also thank R. v. Hees for the provision of the NADC library and related support. This work has been funded by DLR-Bonn and by the University of Bremen.

Edited by: J.-P. Pommereau

\section{References}

Bingen, C. and Fussen, D.: Structure and spectral features of the stratospheric aerosol extinction profiles in the UV-visible range derived from SAGE II data, J. Geophys. Res., 105, 4767-4776, doi:10.1029/1999JD901109, 2000.

Bogumil, K., Orphal, J., Homann, T., Voigt, S., Spietz, P., Fleischmann, O. C., Vogel, A., Hartmann, M., Bovensmann, H.,

\footnotetext{
${ }^{5}$ This equation is for PMDs 1-5. For PMD 7, exchange $\mu_{2}^{\mathrm{P}} \rightarrow$ $\mu_{3}^{\mathrm{P}}$ and $q^{\mathrm{S}} \rightarrow u^{\mathrm{S}}$.
}

Frerik, J., and Burrows, J. P.: Measurements of molecular absorption spectra with the SCIAMACHY pre-flight model: instrument characterization and reference data for atmospheric remote sensing in the 230-2380 nm region, J. Photochem. Photobiol. A, 157, 167-184, doi:10.1016/S0010-4655(02)00555-6, 2003.

Bolle, H. (Ed.): A Preliminary Cloudless Standard Atmosphere for Radiation Computation, vol. WCP-112, World Climate Research Program, World Meteorological Organization, Geneva, Switzerland, 1986.

Bovensmann, H., Burrows, J. P., Buchwitz, M., Frerick, J., Noël, S., Rozanov, V. V., Chance, K. V., and Goede, A. H. P.: SCIAMACHY - mission objectives and measurement modes, J. Atmos. Sci., 56, 127-150, 1999.

Bramstedt, K., Noël, S., Bovensmann, H., Burrows, J. P., Lerot, C., Tilstra, L. G., Lichtenberg, G., Dehn, A., and Fehr, T.: SCIAMACHY monitoring factors: Observation and end-to-end correction of instrument performance degradation, in: Proc. Atmos. Sci. Conf., Barcelona, Spain, 7-11 September 2009 (ESA SP676), ESA, Nordwijk, Netherlands, 2009.

Bréon, F., Buriez, J., Couvert, P., Deschamps, P., Deuzé, J., Herman, M., Goloub, P., Leroy, M., Lifermann, A., Moulin, C., Parol, F., Sèze, G., Tanré, D., Vanbauce, C., and Vesperini, M.: Scientific results from the Polarization and Directionality of the Earth's Reflectances (POLDER), Adv. Space Res., 30, 2383 2386, doi:10.1016/S0273-1177(02)80282-4, 2002.

Burrows, J. P., Weber, M., Vladimir Rozanov, M. B., LadstätterWeißenmayer, A., Richter, A., DeBeek, R., Hoogen, R., Bramstedt, K., Eichmann, K.-U., Eisinger, M., and Pernerb, D.: The Global Ozone Monitoring Experiment (GOME): Mission concept and first results, J. Atmos. Sci., 56, 151-175, 1999.

Callies, J., Corpaccioli, E., Eisinger, M., Lefebvre, A., Munro, R., and Perez-Albinana, A.: New advanced polarization measurements of GOME-2 onboard the Metop satellites, Proc. SPIE, 4814, 131-141, 2002.

COESA: US Standard Atmosphere, Committee on Extension to the Standard Atmosphere, Government Printing Office, Washington, DC, 1976.

Deepak, A. and Gerber, H. E. (Eds.): Report on the experts meeting on aerosols and their climatic effects, vol. WCP-55, World Climate Program, World Meteorological Organization, Geneva, Switzerland, 1983.

Deschamps, P.-Y., Breon, F.-M., Leroy, M., Podaire, A., Bricaud, A., Buriez, J.-C., and Seze, G.: The POLDER mission: instrument characteristics and scientific objectives, IEEE T. Geosci. Remote, 32, 598-615, doi:10.1109/36.297978, 1994.

Emde, C., Buras, R., Mayer, B., and Blumthaler, M.: The impact of aerosols on polarized sky radiance: model development, validation, and applications, Atmos. Chem. Phys., 10, 383-396, doi:10.5194/acp-10-383-2010, 2010.

Frerick, J.: How to generate phase shift corrected greek key data, Technical Note IFE-TN-211299, IfE, 1999.

Gottwald, M. and Bovensmann, H.: SCIAMACHY - Exploring the Changing Earth's Atmosphere, Springer, Dordrecht Heidelberg London New York, doi:10.1007/978-90-481-9896-2, 2011.

Haley, C. S., Brohede, S. M., Sioris, C. E., Griffioen, E. Murtagh, D. P., McDade, I. C., Eriksson, P., Llewellyn, E. J., Bazureau, A., and Goutail, F.: Retrieval of stratospheric $\mathrm{O}_{3}$ and $\mathrm{NO}_{2}$ profiles from Odin Optical Spectrograph and Infrared Imager System (OSIRIS) limb-scattered sunlight measurements, J. 
Geophys. Res., 109, D16303, doi:10.1029/2004JD004588, 2004.

Hansen, J. E. and Travis, L. D.: Light scattering in planetary atmospheres, Space Sci. Rev., 16, 527-610, 1974.

Kokhanovsky, A., Breon, F.-M., Cacciari, A., Carboni, E., Diner, D., Nicolantonio, W. D., Grainger, R., Grey, W., Höller, R., Lee, K.H., Li, Z., North, P., Sayer, A., Thomas, G., and von HoyningenHuene, W.: Aerosol remote sensing over land: a comparison of satellite retrievals using different algorithms and instruments, Atmos. Res., 85, 372-394, doi:10.1016/j.atmosres.2007.02.008, 2007.

Krijger, J. M., Tanzi, C. P., Aben, I., and Paul, F.: Validation of GOME polarization measurements by method of limiting atmospheres, J. Geophys. Res., 110, D07305, doi:10.1029/2004JD005184, 2004.

Krijger, J. M., Snel, R., and Slijkhuis, S.: Revised polarisation calibration of SCIAMACHY, in: Proc. Atmos. Sci. Conf., Barcelona, Spain, 7-11 September 2009 (ESA SP-676), ESA, Nordwijk, Netherlands, 2009.

Lebsock, M., L'Ecuyer, T. S., and Stephens, G. L.: Information content of near-infrared spaceborne multiangular polarization measurements for aerosol retrievals, J. Geophys. Res., 112, D14206, doi:10.1029/2007JD008535, 2007.

Mayer, B.: Radiative transfer in the cloudy atmosphere, EPJ Web of Conferences, 1, 75-99, doi:10.1140/epjconf/e2009-00912-1, 2009.

McLinden, C. A., McConnell, J. C., McElroy, C. T., and Griffioen, E.: Observations of stratospheric aerosol using CPFM polarized limb radiances, J. Atmos. Sci., 56, 233-240, doi:10.1175/1520-0469(1999)056<0233:OOSAUC > 2.0.CO;2, 1999.

McLinden, C. A., Haley, C. S., and Llewellyn, E. J.: Derivation of polarization from Odin/OSIRIS limb spectra, Geophys. Res. Lett., 31, L20112, doi:10.1029/2004GL020825, 2004.

McLinden, C. A., Haley, C. S., Lloyd, N. D., Hendrick, F., Rozanov, A., Sinnhuber, B.-M., Goutail, F., Degenstein, D. A., Llewellyn, E. J., Sioris, C. E., Roozendael, M. V., Pommereau, J. P., Lotz, W., and Burrows, J. P.: Odin/OSIRIS observations of stratospheric BrO: Retrieval methodology, climatology, and inferred $\mathrm{Br}_{\mathrm{y}}$, J. Geophys. Res., 115, D15308, doi:10.1029/2009JD012488, 2010.

Munro, R., Eisinger, M., Anderson, C., Callies, J., Corpaccioli, E., Lang, R., Lefebvre, A., Livschitz, Y., and Albiñana, A. P.: GOME-2 on MetOp, in: Proc. of The 2006 EUMETSAT Meteorological Satellite Conference, Helsinki, Finland, 1216 June 2006, EUMETSAT P.48, EUMETSAT, Darmstadt, Germany, 2006.

Oikarinen, L., Sihvola, E., and Kyrölä, E.: Multiple scattering radiance in limb-viewing geometry, J. Geophys. Res., 104, 3126131274, doi:10.1029/1999JD900969, 1999.

Rothman, L., Jacquemart, D., Barbe, A., Benner, D. C., Birk, M., Brown, L., Carleer, M., Chackerian Jr., C., Chance, K., Coudert, L., Dana, V., Devi, V., Flaud, J.-M., Gamache, R., Goldman, A., Hartmann, J.-M., Jucks, K., Maki, A., Mandin, J.-Y., Massie, S., Orphal, J., Perrin, A., Rinsland, C., Smith, M., Tennyson, J., Tolchenov, R., Toth, R., Auwera, J. V., Varanasi, P., and Wagner, G.: The HITRAN 2004 molecular spectroscopic database, J. Quant. Spectrosc. Ra., 96, 139-204, doi:10.1016/j.jqsrt.2004.10.008, 2005.
Rozanov, A. V., Rozanov, V. V., and Burrows, J. P.: Combined differential-integral approach for the radiation field computation in a spherical shell atmosphere: nonlimb geometry, J. Geophys. Res., 105, 22937-22942, doi:10.1029/2000JD900378, 2000.

Rozanov, A. V., Rozanov, V. V., and Burrows, J.: A numerical radiative transfer model for a spherical planetary atmosphere: combined differential-integral approach involving the Picard iterative approximation, J. Quant. Spectrosc. Ra., 69, 491-512, doi:10.1016/S0022-4073(00)00100-X, 2001.

Rozanov, A. V., Rozanov, V. V., and Burrows, J. P.: Evaluation of ther combined differential-integral approach for limb viewing geometry, Adv. Space Res., 29, 1843-1848, 2002.

Rozanov, V. V., Rozanov, A. V., Kokhanovsky, A. A., and Burrows, J. P.: Radiative transfer through terrestrial atmosphere and ocean: software package SCIATRAN, J. Quant. Spectr. Ra., accepted, 2013.

Schutgens, N. A. J., Tilstra, L. G., Stammes, P., and Breon, F.-M.: On the relationship between Stokes parameters $Q$ and $U$ of atmospheric ultraviolet/visible/near-infrared radiation, J. Geophys. Res., 109, D09205, doi:10.1029/2003JD004081, 2004.

Slijkhuis, S.: ENVISAT-1 SCIAMACHY Level 0 to 1c Processing Algorithm Theoretical Basis Document, ENVATB-DLR-SCIA-0041 Issue 5, DLR, http://atmos.caf.dlr. de/sciamachy/documents/level_0_1b/atbd_101_ipf7.pdf, (last access: 20 May 2008), 2008.

Snel, R.: SCIAMACHY on-ground performance: polarisation phase shift, in: Proceedings of ESAMS'99 - European Symposium on Atmospheric Measurements from Space, ESTEC, Nordwijk, The netherlands, 18-22 January 1999 (WPP 161, Vol. 2), 725-729, ESA, Nordwijk, Netherlands, 1999.

Snel, R. and Krijger, J.: An Improved Scanner Model for Sciamachy, in: Proc. Atmos. Sci. Conf., Barcelona, Spain, 7-11 September 2009 (ESA SP-676), ESA, Nordwijk, Netherlands, 2009.

Tilstra, L. G. and Stammes, P.: Earth reflectance and polarization intercomparison between SCIAMACHY onboard Envisat and POLDER onboard ADEOS-2, J. Geophys. Res., 112, D11304, doi:10.1029/2006JD007713, 2007.

Tilstra, L. G. and Stammes, P.: SCIAMACHY Level-1 V7.03: Earth Reflectance and Polarisation, KNMI-RP-2010-01, KNMI, http:// www.knmi.nl/ tilstra/Reports/KNMI-RP-2010-01.pdf, (last access: 9 June 2010) 2010.

van Hees, R. M.: Reference Manual of the nadc-tools API and Libraries, SRON, http://www.sron.nl/ richardh/SciaDC/nadc_libs. pdf, (last access: 18 January 2012), 2012.

Winker, D. M., Vaughan, M. A., Omar, A., Hu, Y., Powell, K. A., Liu, Z., Hunt, W. H., and Young, S. A.: Overview of the CALIPSO Mission and CALIOP Data Processing Algorithms, J. Atmos. Ocean. Tech., 26, 2310, doi:10.1175/2009JTECHA1281.1, 2009. 